\title{
THE GRAPHS WITH ALL SUBGRAPHS T-PERFECT*
}

\author{
A. M. H. GERARDS ${ }^{\dagger}$ AND F. B. SHEPHERD ${ }^{\ddagger}$
}

Abstract. The richest class of t-perfect graphs known so far consists of the graphs with no so-called odd- $K_{4}$. Clearly, these graphs have the special property that they are hereditary $t$-perfect in the sense that every subgraph is also t-perfect, but they are not the only ones. In this paper we characterize hereditary $t$-perfect graphs by showing that any non-t-perfect graph contains a non-tperfect subdivision of $K_{4}$, called a bad-K $K_{4}$. To prove the result we show which "weakly 3-connected" graphs contain no bad- $K_{4}$; as a side-product of this we get a polynomial time recognition algorithm.

It should be noted that our result does not characterize t-perfection, as that is not maintained when taking subgraphs but only when taking induced subgraphs.

AMS subject classifications. $05 \mathrm{C} 75,05 \mathrm{C} 70,90 \mathrm{C} 10,90 \mathrm{C} 27$

Key words. stable sets, polyhedra, odd circuits, decomposition

PII. S0895480196306361

1. Introduction. A graph $G=(V, E)$ is $t$-perfect if the polyhedron

$$
\begin{aligned}
& \mathcal{P}(G):=\left\{x \in \mathbb{R}^{V} \mid \quad x_{v} \geq 0 \quad(v \in V),\right. \\
& \begin{aligned}
x_{u}+x_{v} & \leq 1 & & (u v \in E), \\
\sum_{v \in V(C)} x_{v} & \leq \frac{|V(C)|-1}{2} & & (C \text { is odd circuit in } G)
\end{aligned}
\end{aligned}
$$

has integral vertices only, i.e., when $\mathcal{P}(G)$ is the stable set polytope of $G$. T-perfection was introduced by Chvátal [4], and a characterization of it has proved elusive. The first two classes of graphs known to be t-perfect are series-parallel graphs (conjectured by Chvátal [4] and proved by Boulala and Uhry [2]) and almost bipartite graphs, i.e., graphs with a node that is contained in every odd circuit [5]. A common extension of these two classes is the class of graphs that do not contain an odd- $K_{4}$ as a subgraph. Here odd-K $K_{4}$ means a subdivision of $K_{4}$, the complete graph on four nodes, in which all triangles have become odd circuits (cf. Figure 1a). Graphs containing no odd- $K_{4}$ are t-perfect [9]. However, there are odd $-K_{4}$ 's that are t-perfect, namely, the good- $K_{4}$ 's: a good- $K_{4}$ is a subdivision of $K_{4}$, in which two nonadjacent edges are not subdivided and the other four edges have become even paths (cf. Figure $1 \mathrm{~b}$ ). An odd- $K_{4}$ that is not good is called a bad- $K_{4}$; bad- $K_{4}$ 's are not t-perfect (Lemma 11). The main result of this paper is the following theorem.

THEOREM 1. If $G$ contains no bad- $K_{4}$ as a subgraph, then it is t-perfect.

We prove this in section 3 . One of the main tools is the following decomposition result.

THEOREM 2. If $G$ is weakly 3-connected, i.e., a subdivision of a 3-node-connected simple graph, then it contains no bad-K $K_{4}$ if and only if one of the following holds:

- $G$ contains no odd- $K_{4}$;

- $G$ is an odd- $P_{9}$;

${ }^{*}$ Received by the editors June 12, 1996; accepted for publication (in revised form) November 14 1997; published electronically September 1, 1998. This research was partially supported by project HCM-DONET ERBCHRXCT930090 of the European Community.

http://www.siam.org/journals/sidma/11-4/30636.html

†CWI, P.O. Box 94079, 1090 GB Amsterdam, The Netherlands (bgerards@cwi.nl).

$\ddagger$ Centre for Discrete and Applicable Mathematics, London School of Economics, London WC2A 2AE, UK (bshep@lse.ac.uk). 


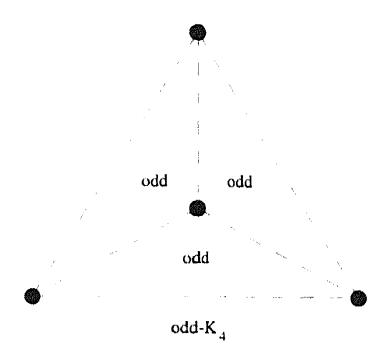

(a)

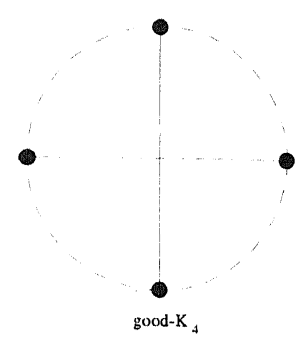

(b)

FIG. 1. Dashed curves indicate internally node disjoint paths of positive length, which in (b) all have even length.

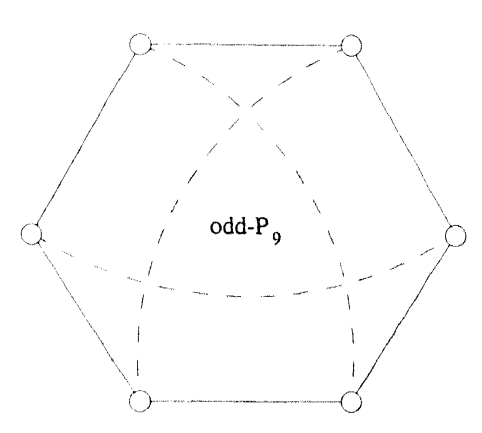

(a)

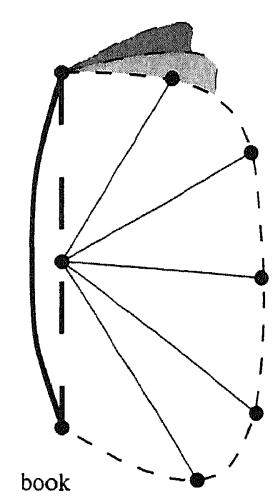

(b)

FIG. 2. Dashed curves indicate internally node disjoint paths of positive even length. The shaded regions in (b) indicate the second and third leaf of the book.

- $G$ is a clean pad;

- $G$ is a book.

An odd- $P_{9}$ is a graph obtained from a six circuit $u_{1} u_{2}, \ldots, u_{5} u_{6}, u_{6} u_{1}$ by adding three node disjoint even $u_{i} u_{i+3}$-paths $(i=1,2,3)$; see Figure $2 a$. Note that the smallest odd $-P_{9}$ is the Petersen graph with a node removed.

A pad is a graph $G$ with a Hamiltonian circuit $w_{1}, u_{1}, w_{2}, u_{2}, \ldots, w_{k}, u_{k}$ such that an edge not on the Hamiltonian circuit has both end nodes in $U(G):=\left\{u_{1}, u_{2}, \ldots, u_{k}\right\}$. (We also define $W(G):=\left\{w_{1}, w_{2}, \ldots, w_{k}\right\}$.)

Clearly, a pad has exactly one Hamiltonian circuit, which we denote by $R(G)$ and call the rim of the pad. The set of edges not on the rim, called chords, will be denoted by $K(G)$. A pad $G$ is clean if neither of the two pads in Figure 3 can be derived from $G$ by deleting chords and contracting edges on the rim.

A book is any graph that can be constructed as follows:

- Take two nodes $h_{1}$ and $h_{2}$ (the hinges of the book), and join them by an edge.

- Take a third node $c$, the center of the book, and add two internally node disjoint even paths, one from $c$ to $h_{1}$ and one from $c$ to $h_{2}$ (together with $h_{1} h_{2}$ these paths form the spine of the book).

- Add $n$ internally node disjoint even $h_{1} h_{2}$-paths $P_{1}, \ldots, P_{n}$, and select on each $P_{i}$ a nonempty collection $T_{i}$ of nodes that are an even distance from $h_{1}$ on $P_{i}$. 

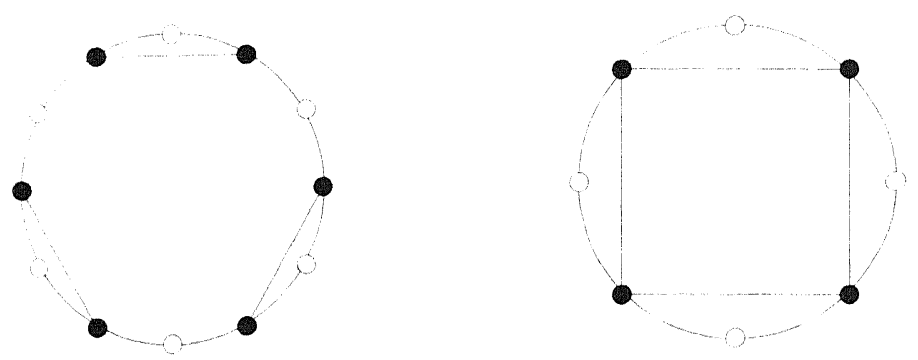

FIG. 3 .

FIG. 4.

- Finally, add all edges in $R_{i}:=\left\{c r \mid r \in T_{i}\right\}, i=1, \ldots, n$.

Note that the union of each $P_{i} \cup R_{i}$ with the spine forms a pad. We call these pads the leaves of the book. The path $P_{i}$ is called the trim of the leaf. Figure $2 \mathrm{~b}$ indicates a book with 3 leaves.

As side-product we obtain the following result (we shall give the easy proof in section 2.3).

THEOREM 3. There exists a polynomial time algorithm that decides whether or not a given graph $G$ contains a bad- $K_{4}$.

Another easy side-product, of which we skip the proof, is that graphs with no bad- $K_{4}$ are 3-colorable. This generalizes a result of Catlin [3] that graphs with no odd $-K_{4}$ are 3 -colorable. Toft [12] conjectures that a graph is 3-colorable if it does not contain a subgraph isomorphic to a graph obtained from $K_{4}$ by replacing all six edges with odd paths.

Characterizations around t-perfection. Shepherd [11] characterized which near-bipartite graphs are t-perfect. (A graph is near-bipartite if for each node $v$ and each odd circuit $C$ there is a neighbor of $v$ on $C$. In fact, Shepherd [11] characterized the stable set polytopes of all near-bipartite graphs.) However, the characterization of t-perfection among all graphs is still open.

The graph in Figure 4 is t-perfect-as is easily proved-but contains a bad- $K_{4}$, which is not t-perfect. Thus t-perfection is not closed under taking subgraphs. Tperfection is however closed under taking induced subgraphs, i.e., under the deletion of nodes, but a complete list of minimally induced non-t-perfect graphs is not yet known.

However, combining Theorem 1 and Lemma 11, we do have the following:

(2) A graph contains no bad- $K_{4}$ if and only if all its subgraphs are t-perfect.

The result of Gerards and Schrijver shows that graphs with no odd- $K_{4}$ are t-perfect. In fact, there it is proved that a graph $G=(V, E)$ has no odd- $K_{4}$ if and only if for all $a, b \in \mathbb{Z}^{V}$ and all $c, d \in \mathbb{Z}^{E}$ the polyhedron 


$$
\left\{x \in \mathbb{R}^{V} \mid a_{v} \leq x_{v} \leq b_{v}(v \in V) ; c_{u v} \leq x_{u}+x_{v} \leq d_{u v}(u v \in E)\right\}
$$

has Chvátal-rank 1, which means that the convex hull of the integral vectors in that polyhedron is obtained by adding all rank-1 Chvátal-Gomory cuts. From Theorem 1 it is not hard to see that a similar result holds for graphs with no bad- $K_{4}$.

COROLlaRY 4. $G=(V, E)$ contains no bad-K $K_{4}$ if and only if for all $a, b \in \mathbb{Z}^{V}$ and all $c \in \mathbb{Z}^{E}$ the polyhedron

$$
\left\{x \in \mathbb{R}^{V} \mid a_{v} \leq x_{v} \leq b_{v}(v \in V) ; x_{u}+x_{v} \leq c_{u v}(u v \in E)\right\}
$$

has Chvátal-rank 1.

The rank-1 Chvátal-Gomory cuts needed here are

$$
\sum_{v \in V(C)} x_{v} \leq \frac{1}{2}\left\lfloor\sum_{u v \in E(C)} c_{u v}\right\rfloor(C \text { is an odd circuit in } G) .
$$

One of the main open questions about t-perfection is whether the system of linear inequalities given in (1) is totally dual integral. This property holds for graphs with no odd- $K_{4}[6]$, but we have not yet been able to verify this for graphs with no bad- $K_{4}$. By the decomposition results used in Gerards [6], it follows that to check for which graphs the system in (1) is totally dual integral for all subgraphs, we may confine ourselves to clean pads and books.

Preliminaries. If $G$ is a graph and $u$ and $v$ are nodes in $G$ of degree at least 3 , then a $u v$-leg of $G$ is a $u v$-path $P$ in $G$ such that all nodes of $P$, except $u$ and $v$, have degree 2 in $G$.

If $P$ is a path in $G$ and $u, v \in V(P)$ we denote the $u v$-path in $P$ by $P_{u v}$. If $e=u v \in E(G), P_{e}:=P_{u v}$.

2. Structure of graphs with no bad- $K_{4}$. We first prove that if a weakly 3 -connected graph with no bad- $K_{4}$ contains an odd- $K_{4}$, then it is either an odd- $P_{9}$, a book, or a pad (Lemma 5). Next we prove that a weakly 3-connected pad with no bad- $K_{4}$ is clean (Lemma 6 ). Together these two lemmas prove the only-if direction of the equivalence in Theorem 2. As odd- $P_{9}$ 's clearly have no bad- $K_{4}$, the if direction follows by proving that clean pads (Lemma 7 ) and books (Lemma 8 ) have no bad- $K_{4}$. We conclude this section with a recognition algorithm for graphs with no bad- $K_{4}$.

2.1. Books and pads. Let $G$ be a pad. If $H$ is a subgraph of $G$ and not a pad itself, we denote by $K(H)$ the edges in $K(G)$ with both end nodes in $V(H)$.

If $P$ is a path on $R(G)$, we say that chords $e$ and $f$ are nested on $P$, written as $e \succ_{P} f$, if $e, f \in K(P)$ and $P_{f}$ is a subpath of $P_{e}$. Chords $e, f$ of $K(G)$ are nested if they are nested on some path on $R(G)$; if not, $e$ and $f$ cross (notation: $e \times f$ ).

LEMMA 5. Let $G$ be a weakly 3-connected graph with no bad-K $K_{4}$. If $G$ contains an odd-K $K_{4}$, then $G$ is an odd-P, a book or a pad.

Proof. We first give some definitions: Let $H$ be a subgraph of a graph $G$. A route of $H$ or an $H$-route is a uv-path $P$ in $G$ such that $V(P) \cap V(H)=\{u, v\}$ and such that no leg of $H$ contains both $u$ and $v$. We say that nodes $u_{1}, u_{2}$, and $u_{3}$ induce an extended triangle in $H$ if each pair is connected by a leg of $H$. A collection of three 


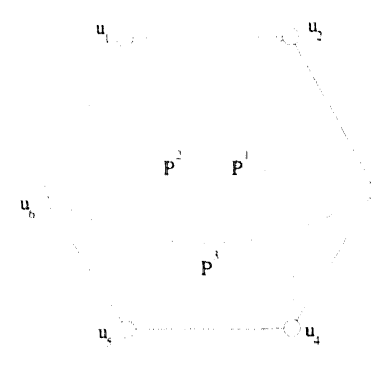

(a)

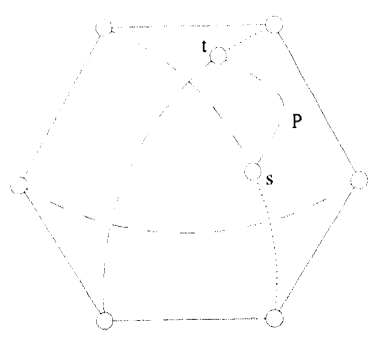

(b)

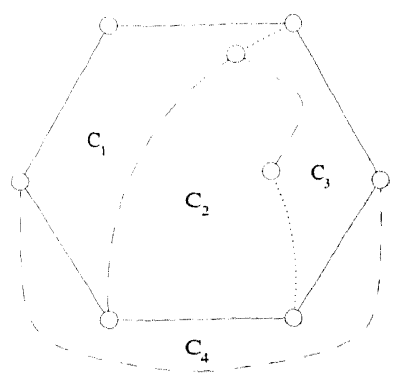

(c)

FIG. 5. Dotted and dashed curves indicate internally node disjoint paths; dashed curves have positive length, whereas dotted curves may have length zero. In (a), dashed curves have an even number of edges.

internally node disjoint $v u_{i}$-paths $P_{i}(i=1,2,3)$ that are internally node disjoint from $H$ is called an $H$-tripod if $v \notin V(H)$ and $u_{1}, u_{2}, u_{3}$ induce an extended triangle in $H$.

It is an easy graph theoretical fact that if $H$ is a weakly 3-connected proper subgraph of a weakly 3 -connected graph $G$, then $G$ contains an $H$-route, or each leg of $H$ is a leg of $G$ and $G$ contains an $H$-tripod. Moreover, adding an $H$-route to a weakly 3-connected graph $H$ yields a weakly 3-connected graph.

Assume that $G$ is a counterexample to the lemma with a minimum number of edges.

Claim 1. G contains no odd-P.

Proof of Claim 1. Suppose the claim is false and that $H$ is an odd- $P_{9}$ in $G$. Let $u_{1} u_{2}, u_{2} u_{3}, \ldots, u_{6} u_{1}$ be the six length-1 legs of $H$; and, for $i=1,2,3$, let $P^{i}$ be the even $u_{i} u_{i+3}$-leg of $H$ (see Figure 5a). By assumption $G \neq H$. As $H$ is weakly 3 -connected and has no extended triangle, there exists an $H$-route $P$ in $G$. Let $s$ and $t$ be the end nodes of $P$. One argues that without loss of generality, $s \in P^{1} \backslash u_{1}$ and $t \in P^{2} \backslash u_{5}$ (see Figure 5b). Let $G^{\prime}:=\left(H \backslash P_{u_{1} s}^{1}\right) \cup P$ and $C_{1}, C_{2}, C_{3}$, and $C_{4}$ be circuits as indicated in Figure 5c. Clearly, $C_{1}$ and $C_{4}$ are odd circuits. Moreover, $C_{2}$ is even, as otherwise the union of $C_{1}, C_{2}$, and $C_{4}$ is a bad- $K_{4}$. Hence, $C_{3}$ is odd, so the union of $C_{4}, C_{3}$, and the symmetric difference of $C_{1}$ and $C_{2}$ forms a bad- $K_{4}$.

Claim 2. If $H$ is a good- $K_{4}$ and $P$ an $H$-route, then $P$ is an edge and $H \cup P$ is a pad with $R(H \cup P)=R(H)$.

Proof of Claim 2. $H$ is a pad. Let $u_{1} u_{3}$ and $u_{2} u_{4}$ be the two chords of $H$ and $Q^{1}, Q^{2}, Q^{3}$ and $Q^{4}$ be the four legs of $H$ on $R(H)$ (see Figure 6a). Let $s$ and $t$ be the two end nodes of $P$. We may assume that $s \in V\left(Q^{1}\right) \backslash\left\{u_{1}, u_{2}\right\}$ and $t \in V\left(Q^{2}\right) \cup V\left(Q^{3}\right) \backslash\left\{u_{2}, u_{4}\right\}$. Let $C$ be the unique circuit in $(R(H) \cup P) \backslash Q^{4}$ (see Figure 6b, c).

First suppose that $C$ is even. If $t$ were in $V\left(Q^{2}\right) \backslash\left\{u_{2}\right\}$ (Figure 6b), then $\left(H \backslash Q_{u_{2} t}^{2}\right) \cup P$ would be an odd- $K_{4}$, with $R_{1}:=u_{1} u_{3}$ and $R_{2}:=u_{4} u_{2} \cup Q_{u_{2} s}^{1}$ as a pair of node disjoint legs. As $R_{1}$ has length 1 and $R_{2}$ does not, this odd- $K_{4}$ would be bad, so $t \in V\left(Q^{3}\right) \backslash\left\{u_{3}, u_{4}\right\}$ (see Figure 6c). As $H \cup P$ is not an odd- $P_{9}$, one of $Q_{u_{1} s}^{1}, Q_{u_{2} s}^{1}, Q_{u_{3} t}^{3}$, and $Q_{u_{4} t}^{3}$ has more than one edge. By symmetry we may assume that this is the case for $Q_{u_{1} s}^{1}$. But then all the legs of the odd- $K_{4} \quad\left(H \backslash Q^{2}\right) \cup P$, except maybe $P$ or $Q_{u_{4} t}^{3}$, have more than one edge. Hence this odd $-K_{4}$ is bad.

Therefore, $C$ is odd and thus $H^{*}:=R(H) \cup P \cup\left\{u_{4} u_{2}\right\}$ is an odd- $K_{4}$. Therefore, $P$ has length 1 and $H^{*}$ is a pad with $R\left(H^{*}\right)=R(H)$. From this it trivially follows 


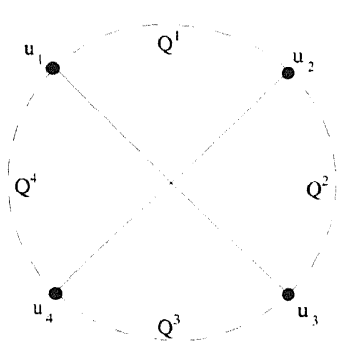

(a)

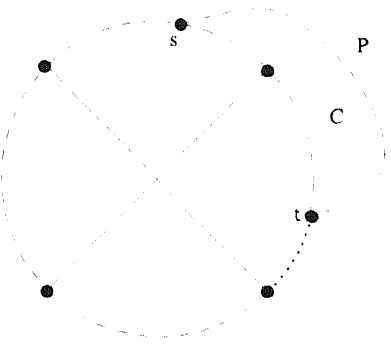

(b)

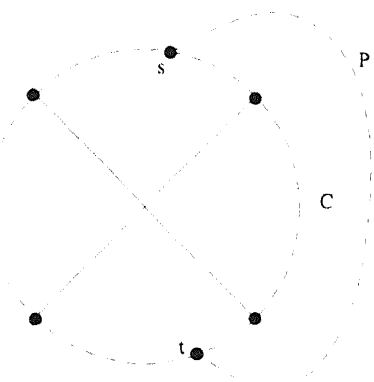

(c)

FIG. 6. Dotted and dashed curves indicate internally node disjoint paths; dashed curves have positive length, whereas dotted curves may have length zero. In (a), dashed curves have an even number of edges.

that also $H \cup P$ is a pad with $R(H \cup P)=R(H)$.

A pad is called maximal if there is no larger pad with the same rim. A subgraph $H$ of $G$ is induced if all edges of $G$ with both end nodes in $V(H)$ are in $H$.

ClaIm 3. No weakly 3-connected maximal pad has a route; hence each one is an induced subgraph of $G$ and has a tripod.

Proof of Claim 3. Let $H$ be a weakly 3-connected pad, and let $P$ be an $H$-route with end nodes $s$ and $t$. Let $Q_{1}$ and $Q_{2}$ be the two st-paths on $R(H)$. As $H^{\prime}:=H \cup P$ is weakly 3-connected, there exists a chord $e=u_{1} u_{2}$ of $H$ with $u_{i} \in V\left(Q_{i}\right) \backslash\{s, t\}$ for $i=1,2$. Moreover, as $H$ is weakly 3 -connected, there exists a chord $f$ of $H$ crossing $e$. Now, $H^{*}:=R(H) \cup\{e, f\}$ is a good- $K_{4}$. As $P$ is an $H$-route, $f \neq s t$. Thus, $s$ and $t$ lie in different legs of $H^{*}$. Hence, by Claim $2, P$ is an edge- $e^{*}$, say-and $H^{*} \cup e^{*}$ is a pad with $R\left(H^{*} \cup e^{*}\right)=R\left(H^{*}\right)=R(H)$. It is trivial to see from this that $H^{\prime}=H \cup e^{*}$ is a pad as well. Hence $H$ is not maximal. Therefore, weakly 3 -connected maximal pads have no routes.

Now, let $H$ be a weakly 3-connected maximal pad. As it is not equal to $G$, it must have a tripod. Moreover, if it were not induced, one of its legs would not be a leg of $G$, but then there would be an $H$-route. As we have seen, this is not the case, so $H$ is an induced subgraph of $G$.

If $H$ is a pad, $u \in V(H)$ is called a center of $H$ if the following hold: $H$ has a chord $v w$ such that all other chords cross it and have $u$ as end node, and $H$ has a tripod such that (i) one of its three paths has end node $u$ and this path is of length 1 and (ii) the other two paths end in $v$ and $w$ and are even. We call such a tripod fitting $H$ at $u$.

Claim 4. Each weakly 3-connected maximal pad has at least one center, and each of its tripods fits at some center of the pad.

Proof of Claim 4. Let $H$ be a weakly 3-connected maximal pad; let $P_{1}, P_{2}$, and $P_{3}$ be the legs of any $H$-tripod. Denote the end node of $P_{i}$ on $H$ by $u_{i}$. Let $Q^{i j}$ be the $u_{i} u_{j}$-path on $R(H)$ that does not contain the third node in $\left\{u_{1}, u_{2}, u_{3}\right\}$. As $H$ is weakly 3-connected, one of $Q^{12}, Q^{23}$, and $Q^{31}$ is not a leg of $H$; i.e., one of the legs of the extended triangle induced by $u_{1}, u_{2}, u_{3}$ is an edge of $K(G)$. Suppose that $Q^{13}$ is not a leg and, consequently, $u_{1} u_{3} \in K(H)$.

$$
\text { If } u_{i} u_{j} \in \mathrm{K}(\mathrm{H}) \text {, then } P_{i} \cup P_{j} \text { is an even path. }
$$

Indeed, if not then $R(H), P_{i} \cup P_{j}$ and one of the chords of $H$ crossing $u_{i} u_{j}$ form a 

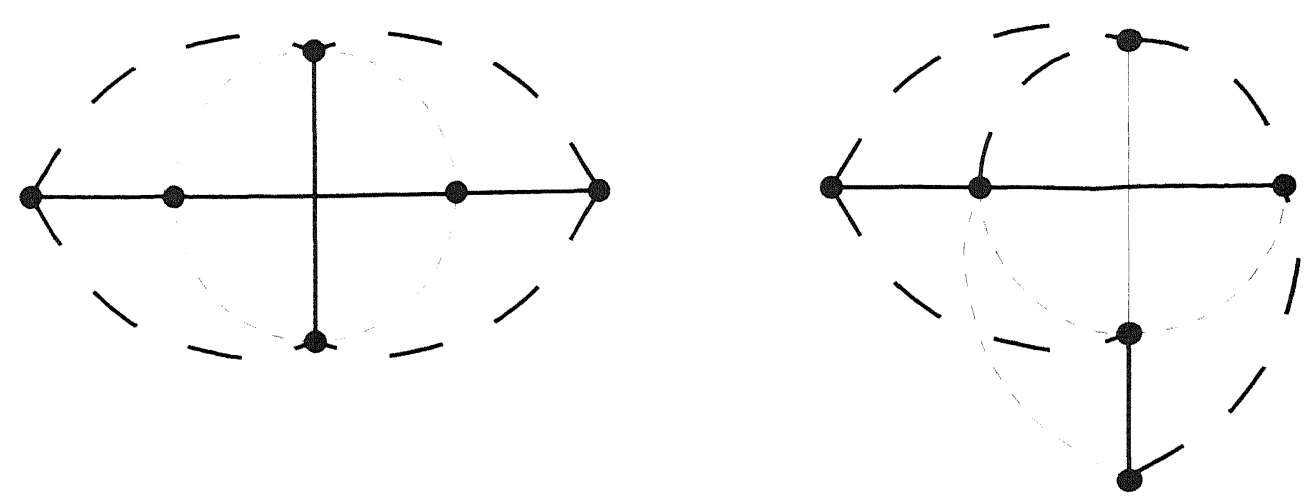

FIG. 7. Dotted curves indicate internally node disjoint even paths. The bold edges and curves form a bad- $K_{4}$.

$\operatorname{bad}-K_{4}$.

(7) $P_{1} \cup P_{2}$ and $P_{2} \cup P_{3}$ are odd paths, and so, by (6), $Q^{12}$ and $Q^{23}$ are legs of $H$.

To see this, let $x y$ be a chord of $H$ crossing $u_{1} u_{3}$; assume $x \in Q^{23}$. It follows from (6) that if (7) were false, then $P_{1}, P_{2}, P_{3}, Q_{u_{2} x}^{23}, x y, Q^{13}$, and $u_{1} u_{3}$ would constitute a bad- $K_{4}$. By (6) and (7), $P_{1}, P_{2}, P_{3}, Q^{12}, Q^{23}$, and $u_{1} u_{3}$ form an odd- $K_{4}$, which implies that

$$
P_{2} \text { consists of a single edge. }
$$

It remains to prove that $u_{2}$ is a center of $H$. Suppose that this is not the case; then there exists a chord $e$ of $H$ with both end nodes in $Q^{13}$ (recall that $Q^{12}$ and $Q^{23}$ are legs of $H$ ). But then $P_{1}, P_{2}, P_{3}, Q^{12}, Q^{23}$, and $\left(Q^{13} \backslash Q_{e}^{13}\right) \cup\{e\}$ form a bad- $K_{4}$.

Claim 5. G contains a book with at least two leaves.

Proof of Claim 5. There exists a weakly 3-connected pad (namely, each good- $K_{4}$ is one). As $G$ is not a pad, by Claim 3 there exists a weakly 3 -connected pad with no route and hence has a tripod. This pad and that tripod together form a book with two leaves.

Let $\widetilde{H}$ be a book with center $c$ and hinges $v$ and $w$, maximum number of leaves $L_{1}, \ldots, L_{n}$, and maximum number of edges. Note that for any $i \neq j, L_{i}$ contains an $L_{j}$-tripod centered at $c$. As in the proof of Claim 4, this implies that each chord of $L_{j}$ has one end in $c$ and the other on the trim of $L_{j}$. Moreover, each $V\left(L_{j}\right)$ induces a maximal pad, so by maximality of $\tilde{H}$, each $L_{j}$ is a maximal pad.

Claim 6. There exists no $\widetilde{H}$-tripod.

Proof of Claim 6. Let $T$ be a tripod of $\widetilde{H}$. As all extended triangles are contained in leaves, we may assume that $T$ is a tripod of leaf $L_{1}$. If $T$ fits $L_{1}$ at the center of the book, $\widetilde{H} \cup T$ would be a larger book. Hence $T$ fits $L_{1}$ at a node different from c. However, then $L_{1}$ has two tripods (namely, $T$ and one in $L_{2}$ ) that fit at different nodes of $L_{1}$, so $L_{1}$ has at least two centers, which implies that it is a good- $K_{4}$. There are two possibilities for how the tripods fit at different nodes (see Figure 7). It is not hard to see that in either case, $L_{1} \cup L_{2} \cup T$ contains a bad- $K_{4}$.

As $G$ itself is not a book, $\widetilde{H}$ has a route- $P$, say. Let $x$ and $y$ be the end nodes of $P$. As the leaves of $\widetilde{H}$ are maximal pads, no one contains both $x$ and $y$, so we may assume that $x \in V\left(L_{1}\right) \backslash V\left(L_{2}\right)$ and $y \in V\left(L_{2}\right) \backslash V\left(L_{1}\right)$. 

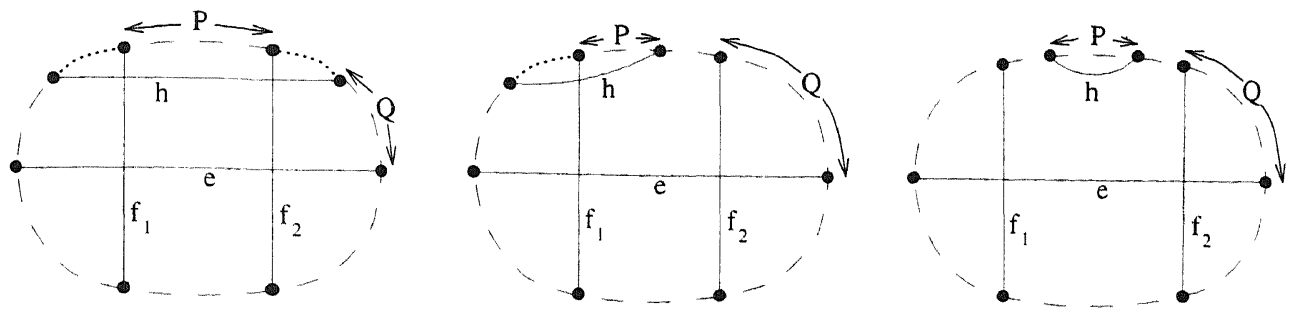

FIG. 8. Dotted and dashed curves indicate internally node disjoint even paths; dashed curves have positive length, whereas dotted curves may have length zero. The closed curve on the outside is the rim.
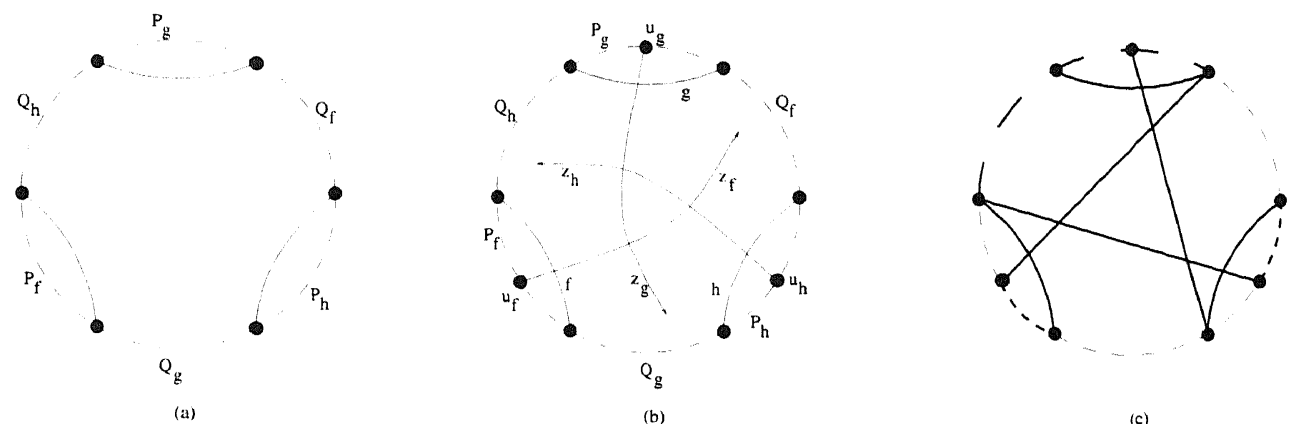

FIG. 9. Dashed curves indicate internally node disjoint even paths of positive length. The closed curve on the outside is the rim.

Let $Q$ be the trim of $L_{2}$. First, if $Q$ and $P$ do not form a tripod of $L_{1}$, then the trim of $L_{1}$ contains at least three legs, so $L_{1}$ has a route, contradicting Claim 3 . Thus $Q$ and $P$ form an $L_{1}$-tripod, which-as $Q$ is even - fits at $x$ (by (7)), so $P$ consists of a single edge and $L_{1}$ has exactly one chord other than $v w$, namely, $x c$. By symmetry, the only chords of $L_{2}$ are $v w$ and $y c$. However, now, $x c, y c, x y$, and the three even paths in $L_{1} \cup L_{2}$ from $v$ to $x, y$, and $c$ form a bad- $K_{4}$. This yields a final contradiction.

2.2. Clean pads. Before we can state and prove the next lemma, we need some further definitions. Let $G$ be a pad. Chords $e$ and $f$ touch, written as $e \vee f$, if they share an end node. Chords $e$ and $f$ are parallel $(e \| f)$ if they are nested but do not touch.

A mesh is a collection of four chords $e, f_{1}, f_{2}, h$ with the following properties:

- $e \times f_{1}, e \times f_{2}, f_{1} \| f_{2}$, and $h \| e$;

- $h$ is not a chord of any of the four legs on $R(G)$ of the pad $R(G) \cup\left\{e, f_{1}, f_{2}\right\}$ that are adjacent with $e$.

There are several possibilities for four chords to form a mesh. They are listed in Figure 8. If we delete the paths $P$ and $Q$ on $R(G)$ indicated in Figure 8, we obtain a bad- $K_{4}$. Hence, a pad with no bad- $K_{4}$ contains no mesh.

A 3-chain is a triple $e, f, g \in K(G)$ such that $e \succ_{P} f \succ_{P} g$ for some path $P$ on $R(G)$. A dirty triple is a collection of three pairwise parallel edges that do not form a 3 -chain (see Figure 9a). A path $P$ on $R(G)$ is nesting if each pair of chords on $K(P)$ is nested. $G$ is nesting if, for each pair of nodes $s, t \in V(G)$, one of the two st-paths on $R(G)$ is nesting.

It is straightforward to prove that 


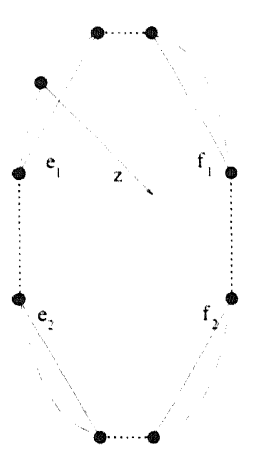

(a)

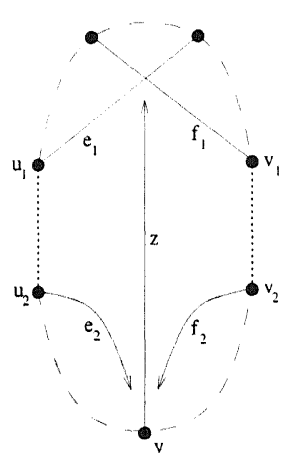

(b)

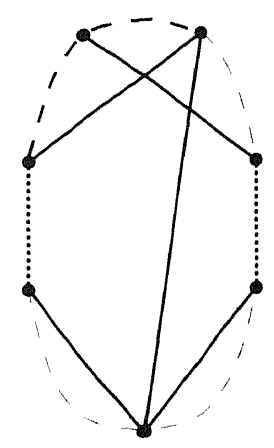

(c)

FIG. 10. Dotted and dashed curves indicate internally node disjoint even paths; dashed curves have positive length, whereas dotted curves may have length zero. The closed curve on the outside is the rim. The bold edges and curves in (c) form a test.

(9) a pad is clean if and only if it is nesting and contains no mesh and no dirty triple.

Lemma 6. Each weakly 3-connected pad with no bad-K $K_{4}$ is clean.

Proof. Let $G$ be a weakly 3 -connected pad with no bad- $K_{4}$. We have already seen that $G$ contains no mesh. Assume that $G$ is not clean.

ClaIM 7. $G$ is nonnesting.

Proof of Claim 7. Suppose that $G$ is nesting. Hence, it contains a dirty triple $T:=\{f, g, h\}$. Let $P_{e}, Q_{e}(e \in T)$ be as in Figure 9a. As $G$ is weakly 3-connected, for each $e \in T$ there exists an edge $z_{e}:=u_{e} v_{e}$ crossing $e$. Assume $u_{e} \in P_{e}$ for each $e \in T$. Then, for each $e \in T, v_{e} \in Q_{e}$, because if $v_{f}$, say, were not in $Q_{f}$, then $z_{f}, g, f, h$ would form a mesh or $G$ would be nonnesting.

By symmetry, we may assume that $z_{f} \| h$. As $z_{f}, z_{h}, f, h$ is no mesh, $z_{h} \vee f$, so $z_{h} \| g$. Repeating this argument we get that $z_{g} \vee h$ and $z_{f} \vee g$. However, now $G$ contains a bad- $K_{4}$ (namely, the bold lines in Figure $9 \mathrm{c}$ ) - a contradiction!

Claim 8. There exist two edge disjoint paths $P_{1}$ and $P_{2}$ on $R(G)$ and edges $e_{1}, f_{1} \in K\left(P_{1}\right)$ and $e_{2}, f_{2} \in K\left(P_{2}\right)$ such that

(i) $e_{i}$ and $f_{i}$ are not nested on $P_{i}(i=1,2)$,

(ii) both $e_{i}$ and $f_{i}$ share an end node with $P_{i}(i=1,2)$,

(iii) $e_{1} \times f_{1}$.

Proof of Claim 8. By the previous claim, there exist two edge disjoint paths $P_{1}$ and $P_{2}$ on $R(G)$ and chords $e_{1}, e_{2}, f_{1}$, and $f_{2}$ satisfying (i). It is not hard to see that these paths and chords can be chosen to satisfy (ii) as well. If neither $e_{1}$ and $f_{1}$ nor $e_{2}$ and $f_{2}$ are crossing, choose $z$ crossing $e_{1}$ ( $G$ is weakly 3 -connected). With the aid of $z$, it is straightforward to see that either we can find edge disjoint paths $P_{1}$ and $P_{2}$ satisfying (i), (ii), and (iii) or we find a mesh (see Figure 9a). As the latter is impossible, the claim follows.

Choose $P_{1}, P_{2}, e_{1}, f_{1}, e_{2}$, and $f_{2}$ as in the previous claim, with $\left|E\left(P_{1}\right)\right|+\left|E\left(P_{2}\right)\right|$ maximal. Let $u_{i}, v_{i}$ be the end nodes of $P_{i}(i=1,2)$. As $G$ is weakly 3 -connected, there exists a chord $z=u v$ with $v \in P_{2} \backslash\left\{u_{2}, v_{2}\right\}$ and $u \notin P_{2}$. By the maximality of $\left|E\left(P_{1}\right)\right|+\left|E\left(P_{2}\right)\right|, u \in P_{1} \backslash\left\{u_{1}, v_{1}\right\}$ (see Figure 10b).

First, consider the special case in Figure 10c. It contains a bad- $K_{4}$, indicated by the bold edges. However, the general case, as in Figure 10b, can be transformed to 


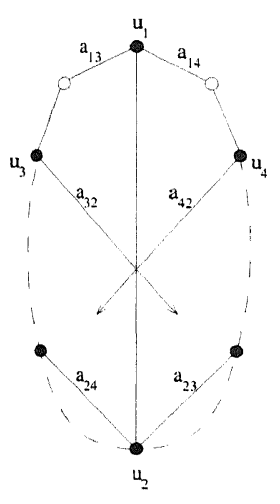

(a)

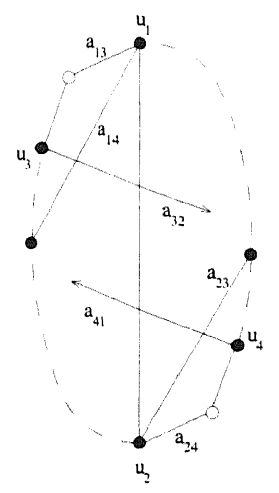

(b)

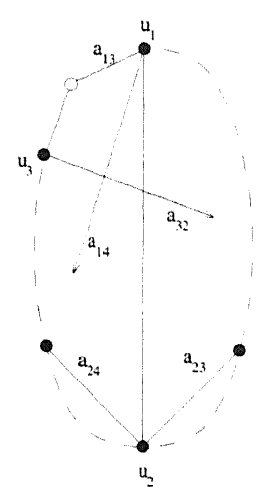

(c)

FIG. 11. Dashed curves indicate internally node disjoint even paths of positive length. The closed curve on the outside is the rim.

that special case by contracting legs on $R(G)$. As legs are even paths, this contraction could not have created a bad- $K_{4}$ if one in $G$ did not already exist. Hence we have a contradiction, so $G$ is clean.

A chord of a pad is called universal if it is not parallel with any other chord.

LEMma 7. No clean pad contains a bad-K $K_{4}$.

Proof. Let $G$ be a clean pad containing a bad- $K_{4} H$ such that $|E(G)|$ is minimal. Let $u_{1}, u_{2}, u_{3}$, and $u_{4}$ be the four nodes of $H$ that have degree three in $H$. For $i, j=1, \ldots, 4$, let $P^{i j}$ be the $u_{i} u_{j}$-leg of $H$.

Claim 9. The following hold:

(i) $K(G) \subseteq E(H)$.

(ii) All legs of $G$ on $R(G)$ have length 2 .

(iii) If $P$ is a leg of $H$, then $|P \cap R(G)| \leq 2$. If $|P \cap R(G)|=2$, then $P$ is a leg of $G$ on $R(G)$ or $P$ has length 3 . In the latter case, the four legs of $H$ meeting $P$ are even, and the sixth leg consists of a single edge.

(iv) If $u, v \in U(G)$ form a 2-node cutset of $G$, then there exists a uv-path $P$ on $R(G)$ with 2 or 4 edges. If $P$ has 4 edges, it has one chord, which meets exactly one of $u$ and $v$.

Proof of Claim 9. If (i) were false, deleting an edge from $K(G) \backslash E(H)$ would contradict the minimality of $G$, as would contracting legs of $G$ into legs of length 2 if (ii) were false.

To prove (iii), suppose $P$ is a leg of $H$ that contains edges of $R(G)$. Let $e_{1}$ and $e_{2}$ be consecutive edges on $P \cap R(G)$. By the minimality of $G$, contracting $e_{1}$ and $e_{2}$ in $H$ does not yield a bad- $K_{4}$. This means that the leg $P$ of $H$ containing $e_{1}$ and $e_{2}$, has length 2 or 3 . Moreover, in the latter case the four legs of $H$ meeting $P$ are even and the sixth one has length one. Hence (iii) follows.

To see (iv), note that if $G$ has a two node cutset, then $H$ lies mainly on one "side" of that cutset in the sense that one side of the cutset contains at least five legs of $H$ and the other side contains at most (part of) the sixth leg.

Claim 10. $G$ has no universal chord.

Proof of Claim 10. Let $u v$ be a universal chord. This means that $G \backslash\{u, v\}$ is bipartite, so $u v$ is a leg of $H$. Assume $u=u_{1}$ and $v=u_{2}$. Let $Q^{1}$ and $Q^{2}$ be the two $u v$-paths in $R(G)$. We call $Q^{1} \cup K\left(Q^{2}\right)$ and $Q^{2} \cup K\left(Q^{1}\right)$ the two sides of $G$. For 
$i, j=1, \ldots, 4$, let $a_{i j}$ be the first edge on $P^{i j}$ going from $u_{i}$ to $u_{j}$. (Thus, $a_{i j}=a_{j i}$ if and only if $\left|P^{i j}\right|=1$.)

As $\left|P^{12}\right|=1$, it follows by Claim 9 that for $i=1,2$ and $j=3,4, a_{i j} \in R(G)$ if and only if $P^{i j}$ is a leg of $G$ in $R(G)$. Moreover, as the circuit $P^{1 i} \cup P^{i 2} \cup\left\{u_{1} u_{2}\right\}$ is odd for $i=3,4$, we have the following:

$$
\begin{aligned}
& \text { If } i=3,4 \text {, then } a_{1 i} \text { and } a_{2 i} \text { lie on the same side of } G \text {. Moreover, } P^{1 i} \text { and } P^{2 i} \\
& \text { are both even or both odd. }
\end{aligned}
$$

Also, as the circuit $P^{i 3} \cup P^{34} \cup P^{4 i}$ is odd for $i=1,2$, we have that

$$
\text { if } i=1,2 \text {, then } a_{i 3} \text { and } a_{i 4} \text { lie on different sides of } G \text {. }
$$

Next we rule out the different cases one by one:

At least one of $a_{13}, a_{14}, a_{23}$, and $a_{24}$ is in $R(G)$.

Suppose that this is not the case and that $a_{13} \in K\left(Q^{1}\right)$. Then from (10) and (11) it follows that $a_{23} \in K\left(Q^{1}\right)$ and $a_{14}, a_{24} \in K\left(Q^{2}\right)$. Thus both $Q^{1}$ and $Q^{2}$ are nonnesting, which is a contradiction.

$$
\text { For } i=1,2 \text {, either } a_{i 3} \text { or } a_{i 4} \text { is in } K(G) \text {. }
$$

To see this, assume that $a_{13} \in Q^{1}$ and $a_{14} \in Q^{2}$ (see Figure 10a). By (10), all legs of $H$ adjacent to $u_{1} u_{2}$ are even. Hence $P^{34}$ is odd, and as $H$ is bad, it has at least three edges. By symmetry, we may assume that $Q_{u_{2} u_{4}}^{2}$ is not internally node disjoint with $P^{34}$. Hence $P^{24} \neq Q_{u_{2} u_{4}}^{2}$. Therefore, by (10), $a_{24} \in K\left(Q^{1}\right)$ and $a_{42} \times u_{1} u_{2}$ (by Claim 9(iii) and since $u_{1} u_{2}$ is universal). However, this implies that $P^{23} \neq Q_{u_{2} u_{3}}^{1}$, so, by (10), $a_{23} \in K\left(Q^{2}\right)$ and $a_{32} \times u_{1} u_{2}$. If $a_{32} \times a_{23}$, then $a_{32}, a_{23}, a_{42}$, and $a_{24}$ form a mesh, so $a_{32}$ and $a_{23}$ do not cross. Similarly, $a_{42}$ and $a_{24}$ do not cross. However, this implies that $G$ is nonnesting, a contradiction. Hence (13) follows.

From the above we may assume that $a_{13} \in Q^{1}$ and $a_{14} \in K\left(Q^{1}\right)$, so $P^{23}$ cannot be $Q_{u_{2} u_{3}}^{1}$. Hence $a_{23} \in K\left(Q^{2}\right)$ and $a_{32} \times u_{1} u_{2}$. First assume that $a_{24} \in Q^{2}$ and consequently $a_{41} \times u_{1} u_{2}$ (see Figure 10b). As $G$ is nesting, by symmetry we may assume that $a_{32} \times a_{23}$, but this implies that $a_{32}, a_{41}, a_{23}$, and $a_{14}$ form a mesh. As $G$ is clean, this is a contradiction, so $a_{24} \notin Q^{2}$. Hence, $a_{24} \in K\left(Q^{1}\right)$ (see Figure 10c). As $a_{32}, a_{41}, a_{23}$, and $a_{24}$ is not a mesh, $a_{32}$ does not cross $a_{23}$. Similarly, $a_{24}$ does not cross $a_{14}$. But this means that $G$ is nonnesting-a contradiction!

A chord is crossed if it is crossed by at least one other chord. We call chords $e_{1}$ and $e_{2}$ distant if $e_{1} \| e_{2}$ and, for $i=1,2$, the path $P_{i}$ on $R(G)$ with the same end nodes as $e_{i}$ but node disjoint from $e_{3-i}$ satisfies $K\left(P_{i}\right)=\left\{e_{i}\right\}$.

ClaIM 11. Each pair of distant chords contains a noncrossed chord.

Proof of Claim 11. Let $e_{1}$ and $e_{2}$ be a pair of distant chords. Suppose that $e_{1}$ is crossed by $z_{1}$ and $e_{2}$ by $z_{2}$. For $i=1,2, z_{i}$ does not cross $e_{3-i}$, as otherwise, $z_{i}$ would be universal, or there would be a mesh, or $e_{1}$ and $e_{2}$ would not be distant. As $G$ is nesting $z_{1} \times z_{2}$. Let $x_{1}$ be the end node of $z_{1}$ and $x_{2}$ be the end node of $z_{2}$ such that there exists an $x_{1} x_{2}$-path on $R(G)$, called $Q$, that is internally node disjoint with $e_{1}$ and $e_{2}$. Assume $z_{1}$ and $z_{2}$ are selected such that $Q$ is as short as possible. As $G$ contains no mesh, either $z_{1} \vee e_{2}$ or $z_{2} \vee e_{1}$; assume the latter is the case (see Figure 11).

For $i=1,2$, let $y_{i}$ be a chord parallel with $z_{i}\left(z_{1}\right.$ and $z_{2}$ are not universal). From the fact that $G$ is clean, that $e_{1}$ and $e_{2}$ are distant, and that $Q$ is minimal, one is able 


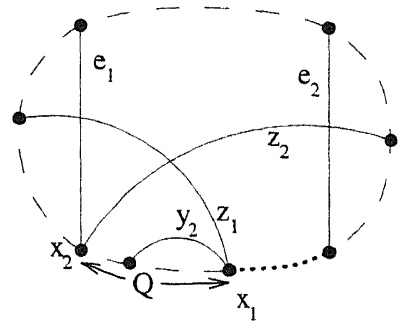

FIG. 12. Dotted and dashed curves indicate internally node disjoint even paths; dashed curves have positive length, whereas dotted curves may have length zero. The closed curve on the outside is the rim.

to deduce that $y_{2} \in K\left(Q \backslash x_{2}\right)$. As $e_{1}, e_{2}, y_{2}$ cannot form a dirty triple, $y_{2}$ is adjacent to $e_{2}$, so $x_{1}$ is an end node of $e_{2}$. Hence we have symmetry between $i=1$ and $i=2$. Therefore, $y_{1} \in K\left(Q \backslash x_{1}\right)$ and is adjacent to $e_{1}$, but now the edges $e_{1}, e_{2}, y_{1}$, and $y_{2}$ show that $G$ is not nesting-a contradiction!

If $e=u v$ is a noncrossed chord, then $u$ and $v$ share a common neighbor in $R(G)$ (by Claim 9(iv)), which we denote by $u_{e}$. As $e \in E(H)$, the node $u_{e}$ will not be in $V(H)$.

ClaIM 12. Each pair of distant chords contains a crossed chord. Moreover, the noncrossed chords in $G$ are pairwise adjacent and there are at most two of them.

Proof of Claim 12. To prove the first statement, suppose that it is false. Let $e$ and $f$ be two parallel nonadjacent noncrossed chords. Let $Q^{1}$ and $Q^{2}$ be the two paths on $R(G)$ joining an end of $e$ with an end of $f$. As $G$ is nesting, we may assume that $K\left(Q^{2}\right)=\emptyset$ and that $Q^{1}$ is nesting. As $H$ is contained in $G^{\prime}=G \backslash\left\{u_{e}, u_{f}\right\}, G^{\prime}$ is nonbipartite. Hence $K\left(Q^{1}\right) \neq \emptyset$. Let $h \in K\left(Q^{1}\right)$ with $Q_{h}^{1}$ minimal. As $G$ has no dirty triple, $h$ is adjacent to $e$ or to $f$. Thus, let us assume that $h$ and $e$ share an end node $-v$, say. As $Q^{1}$ is nesting all chords in $K\left(Q^{1}\right)$ are in $\delta(v)$. But that means that all odd circuits in $G^{\prime}$ contain $v$. This is impossible since not all odd circuits in $H$ can go through a single node.

The second statement easily follows from the first. Indeed, two parallel noncrossed chords are clearly distant by Claim 9(iv), so by the first statement of this claim they cannot exist. Suppose there are three pairwise adjacent noncrossed chords $e_{1}, e_{2}$, and $e_{3}$. They cannot meet at a single node, as this would contradict Claim 9(iv), so they form a triangle. Hence $K(G)=\left\{e_{1}, e_{2}, e_{3}\right\}$ and $R(G)$ is a circuit of length 6 , but that graph has no bad- $K_{4}$.

CLAIM 13. There is exactly one noncrossed chord.

Proof of Claim 13. Suppose that this claim is false. Let $e=x y$ and $f=y z$ be two noncrossed chords. Let $Q$ be the $x z$-path on $R(G)$ not containing $y$. As $e$ is not universal, $K(Q) \neq \emptyset$. Let $g \in K(Q)$ with $Q_{g}$ minimal. Let $h \times g$ (by the previous claim, $g$ is crossed). As $Q$ is nesting, $h \in \delta(y)$ and each chord in $K(Q)$ crosses $h$. Hence, $h$ is universal-a contradiction!

As there are no universal edges, there exists a pair of distant chords. By Claims 11 and 12 one of the two- $e=u v$, say-is crossed, and the other, $f$, is not. Let $P$ be the uv-path on $R(G)$ not containing $u_{f}$. Let $Q^{1}$ and $Q^{2}$ be the two paths constituting $R(G) \backslash\left(P \cup\left\{u_{f}\right\}\right)$. For $i=1,2$, let $K_{i}$ be the collection of edges crossing $e$ with end node in $Q^{i}$.

Claim 14. $K\left(Q^{1}\right)=K\left(Q^{2}\right)=\emptyset, K_{1} \neq \emptyset$, and $K_{2} \neq \emptyset$.

Proof of Claim 14. As $G$ is nesting, (i) $K\left(Q^{1}\right)=\emptyset$ or $K\left(Q^{2}\right)=\emptyset$, (ii) $K\left(Q^{1}\right)=\emptyset$ 
or $K^{2}=\emptyset$, and (iii) $K\left(Q^{2}\right)=\emptyset$ or $K^{1}=\emptyset$. From this it is easy to check that if the claim is false, then either $K^{1}=\emptyset$ and $K\left(Q^{1}\right)=\emptyset$ or $K^{2}=\emptyset$ and $K\left(Q^{2}\right)=\emptyset$. Assume that the latter is the case. Let $w$ be the common end node of $P$ and $Q^{1}$. There exists an odd circuit in $H$ not containing $w$. As $u_{f} \notin V(H)$, this means that $G \backslash\left\{u_{f}, w\right\}$ is nonbipartite. It is straightforward to check that this implies that $K\left(Q^{1}\right)$ contains a chord parallel with $e$. Let $h$ be such a chord with $Q_{h}^{1}$ minimal. Then $e$ and $h$ are distant, so by Claim 11, $h$ is noncrossed, but this contradicts Claim 13.

Let $Q$ be the path $R(G) \backslash u_{f}$. For $i=1,2$, let $e_{i} \in K^{i}$ with $Q_{e_{i}} \cap P$ maximal. As $e, e_{1}, e_{2}, f$ do not from a mesh, $e_{1} \times e_{2}$ or $e_{1} \vee e_{2}$, so there exists a node- $w$, say- that lies on $Q_{e_{1}} \cap P$ and on $Q_{e_{2}} \cap P$. By Claim 14 this means that $w$ lies on $Q_{g}$ for each chord $g \in K(Q)=K(G)$. Hence $G \backslash\left\{w, u_{f}\right\}$ is bipartite. As $H$ does not contain $u_{f}$, this is a final contradiction.

Lemma 8. No book contains a bad-K $K_{4}$.

Proof. Suppose that $G$ is a book, and let $H$ be any odd- $K_{4}$ in $G$. Let $C$ be the spine, $h_{1}$ and $h_{2}$ be the hinges, and $c$ be the center of $G$. It is easy to see that for every $e \in E(C)$ there is a node $v \in\left\{h_{1}, h_{2}, c\right\}$ such that each odd circuit in $G \backslash e$ contains $v$. Hence $H$ must contain $C$. Consequently, $H$ should be entirely contained in one of the leaves of $G$. As all leaves are clean pads, $H$ must be a good- $K_{4}$.

2.3. Recognizing graphs with no bad- $K_{4}$. In this section we prove Theorem 3, which says that one can check-in polynomial time--whether or not a given graph $G$ contains a bad- $K_{4}$.

First of all, note that odd- $P_{9}$ 's, books and clean pads are easily recognized. Second, a polynomial-time recognition algorithm for the containment of an odd- $K_{4}$ is given by Gerards et. al. [8] (cf. Gerards [7]). Hence, by Theorem 2, it suffices to prove that we can find for each graph $G$ in polynomial time a polynomial-length list $\mathcal{L}$ of weakly 3-connected graphs smaller than $G$ such that $G$ contains a bad- $K_{4}$ if and only if at least one member of $\mathcal{L}$ contains a bad- $K_{4}$. The following two easy-to-prove lemmas show that this is indeed the case.

We need some definitions and notations. If $G$ is a graph, then $\left[G_{1}, G_{2}\right]_{u, v}$ is called a split if $G_{1}$ and $G_{2}$ are subgraphs of $G$ such that $V\left(G_{1}\right) \cap V\left(G_{2}\right)=\{u, v\} ; E\left(G_{1}\right)$ and $E\left(G_{2}\right)$ partition $E(G),\left|E\left(G_{1}\right)\right|,\left|E\left(G_{2}\right)\right| \geq 4$; and neither $G_{1}$ nor $G_{2}$ is an odd circuit. If $G_{2}$ is bipartite and contains an odd $u v$-path, we call the split odd. If $G_{2}$ is bipartite and contains an even uv-path, we call the split even. If both $G_{1}$ and $G_{2}$ are nonbipartite, we call the split strong.

If $u$ and $v$ are two nodes of a graph $H$ and $\ell \in \mathbb{N}$, then $[H]_{u, v}^{\ell}$ denotes the graph obtained from $H$ by adding a path from $u$ to $v$ with $\ell$ edges; we abbreviate this as $[H]_{u, v}^{k, \ell}:=\left[[H]_{u, v}^{k}\right]_{u, v}^{\ell}$. hold:

LEMMA 9. Let $\left[G_{1}, G_{2}\right]_{u, v}$ be a split of a 2-connected graph $G$. Then the following

- If $\left[G_{1}, G_{2}\right]_{u, v}$ is odd, then $G$ contains a bad-K $K_{4}$ if and only if $\left[G_{1}\right]_{u, v}^{3}$ contains a bad-K $K_{4}$.

- If $\left[G_{1}, G_{2}\right]_{u, v}$ is even, then $G$ contains a bad-K $K_{4}$ if and only if $\left[G_{1}\right]_{u, v}^{2}$ contains a bad-K $K_{4}$.

- If $\left[G_{1}, G_{2}\right]_{u, v}$ is strong and $G$ has no odd or even split, then $G$ contains a bad-K $K_{4}$ if and only if at least one of $\left[G_{1}\right]_{u, v}^{2,3}$ and $\left[G_{2}\right]_{u, v}^{2,3}$ contains a bad-K $K_{4}$.

It follows from this lemma that given a graph $G$ we can construct a polynomialsized list $\mathcal{L}^{\prime}(G)$ of graphs with no splits such that $G$ has a bad- $K_{4}$ if and only if at least one member of the list has a bad- $K_{4}$. Therefore, we may restrict ourselves to graphs with no split. It is easy to see that a graph with no split can be obtained from 
a 3-connected graph $H$ by replacing some edges in $H$ by a path of length 2 or 3 or by a circuit of length 3 or 5 . More precisely, a graph $G$ has no split if and only if there exists a 3-connected graph $H$ and five sets $P_{1}, P_{2}, P_{3}, C_{3}$, and $C_{5}$ partitioning $E(H)$, such that $G$ can be obtained from $H$ as follows: for each edge $u v \in P_{2} \cup C_{3} \cup C_{5}$ add a path from $u$ to $v$ with 2 edges; moreover, for each edge $u v \in P_{3} \cup C_{5}$ add a path from $u$ to $v$ with 3 edges; finally, remove all the edges in $P_{2} \cup P_{3} \cup C_{5}$. We denote $G$ by $H\left(P_{1}, P_{2}, P_{3}, C_{3}, C_{5}\right)$. Note that, given $G$, it is easy to find $H$ and the proper partition of its edge set.

So we see that a graph with no split only fails to be weakly 3-connected because it may have pairs of "parallel" legs. Clearly, from each such pair of legs a bad- $K_{4}$ can use at most one leg. So if we would consider the list of graphs obtainable by dropping a leg from each pair of parallel ones, we do not gain or lose bad- $K_{4}$ 's. The nice thing about the graphs on this list is that they are weakly 3-connected; the bad thing is that there may be exponentially many of them. Fortunately there is an easy way out of this; we do not need to create the whole list.

LEMMA 10. Let $G=H\left(P_{1}, P_{2}, P_{3}, C_{3}, C_{5}\right)$ be a graph with no split. Then $G$ contains a bad- $K_{4}$ if and only if there exists a $T_{3} \subseteq C_{3}$ and $a T_{5} \subseteq C_{5}$ with $\left|T_{3}\right|+\left|T_{5}\right| \leq$ 6 , such that the graph $H\left(P_{1} \cup T_{3}, P_{2} \cup\left(C_{3} \backslash T_{3}\right) \cup\left(C_{5} \backslash T_{5}\right), P_{3} \cup T_{5}, \emptyset, \emptyset\right)$ contains a bad- $K_{4}$. 3.)

(In fact, this lemma remains correct if we replace $\left|T_{3}\right|+\left|T_{5}\right| \leq 6$ with $\left|T_{3}\right|+\left|T_{5}\right| \leq$

3. T-perfection. The main goal of this section is to prove Theorem 1 , but we first show that bad- $K_{4}$ 's are not t-perfect. In the remainder of the paper, for a subset $S \subseteq V(G)$, we use $\chi_{S}$ to denote the incidence vector of $S$ in $\mathbb{R}^{V(G)}$.

LEMMA 11. No bad-K $K_{4}$ is t-perfect.

Proof. First, note that $K_{4}$ is not t-perfect, as the vector $\left[\frac{1}{3}, \frac{1}{3}, \frac{1}{3}, \frac{1}{3}\right]$ is in $\mathcal{P}\left(K_{4}\right)$, but obviously not the convex combination of characteristic vectors of stable sets in $K_{4}$. Next, note that each bad- $K_{4}$ can be reduced to $K_{4}$ by repeated application of the following operation: take a node $u$ and contract all the edges incident with $u$. However, this operation preserves t-perfection, which we easily obtain from the following:

(14) Let $G$ be a graph, $u \in V(G)$, and $x \in \mathbb{R}^{V(G)}$ such that $x_{v}=1-x_{u}$ for each neighbor $v$ of $u$. Moreover, let $\widetilde{G}$ be obtained from $G$ by contracting all the edges in $\delta(u)$ into a new node $\widetilde{u}$, and let $\widetilde{x} \in \mathbb{R}^{V(\widetilde{G})}$ be defined by $\widetilde{x}_{v}:=x_{v}$ if $v \in V(\widetilde{G}) \backslash \widetilde{u}$ and $\widetilde{x}_{\widetilde{u}}:=1-x_{u}$. Then $x$ is a vertex of $\mathcal{P}(G)$ if and only if $\widetilde{x}$ is a vertex of $\mathcal{P}(\widetilde{G})$.

Hence no bad- $K_{4}$ is t-perfect.

The proof of Theorem 1 uses the following lemma (the graphs $\left[G_{i}\right]_{u, v}^{\ell}$ are defined in section 2.3).

LEMMA 12. Let $G$ be a graph with induced subgraphs $G_{1}$ and $G_{2}$ such that $V(G)=$ $V\left(G_{1}\right) \cup V\left(G_{2}\right)$ and $E(G)=E\left(G_{1}\right) \cup E\left(G_{2}\right)$.

(a) If $V\left(G_{1}\right) \cap V\left(G_{2}\right)$ induces a clique in $G$, then $G$ is t-perfect if and only if $G_{1}$ and $G_{2}$ are t-perfect (Chvátal [4]).

(b) If $G$ is 2-connected, $G_{2}$ is bipartite, and $V\left(G_{1}\right) \cap V\left(G_{2}\right)=\{u, v\}$ with uv $\notin$ $E(G)$, then if $u$ and $v$ are on the same side of the bipartition of $G_{2}, G$ is $t$-perfect if and only if $\left[G_{1}\right]_{u, v}^{2}$ is t-perfect; otherwise, $G$ is t-perfect if and only if $\left[G_{1}\right]_{u, v}^{3}$ is t-perfect (Sbihi and Uhry [10]). 
(c) If $G$ is 2-connected, both $G_{1}$ and $G_{2}$ are nonbipartite, and $V\left(G_{1}\right) \cap V\left(G_{2}\right)=$ $\{u, v\}$ with $u v \notin E(G)$, then $G$ is t-perfect if and only if $\left[G_{1}\right]_{u, v}^{2},\left[G_{1}\right]_{u, v}^{3},\left[G_{2}\right]_{u, v}^{2}$, and $\left[G_{2}\right]_{u, v}^{3}$ are t-perfect (Boulala and Uhry [2], Gerards [6]).

In fact, the lemma above can be generalized beyond t-perfection: It has been proved by Chvátal [4]-for case (a)-and Barahona and Mahjoub [1]-for cases (b) and (c) - that one can obtain linear descriptions for the stable set polyhedron recursively through decompositions as in Lemma 12 .

Proof of Theorem 1. Let $\widetilde{G}$ be a counterexample to the theorem with $|E(\widetilde{G})|$ minimal. By Lemma 12

$$
\widetilde{G} \text { is weakly } 3 \text {-connected and each of its legs has at most } 3 \text { edges. }
$$

Let $\widetilde{x}$ be a fractional vertex of $\mathcal{P}(\widetilde{G})$. An edge $u v \in E(\widetilde{G})$ is tight if $\widetilde{x}_{u}+\widetilde{x}_{v}=1$; an odd circuit $C$ is tight if $\sum_{v \in V(C)} \widetilde{x}_{v}=\frac{1}{2}(|V(C)|-1)$. We denote the collection of tight edges by $\mathcal{T}$ and the collection of tight odd circuits by $\mathcal{C}$.

$$
0<\widetilde{x}_{v}<1 \text { for each } v \in V(\widetilde{G}) .
$$

Indeed, if $\widetilde{x}_{u}=0$, then $\widetilde{G} \backslash\{u\}$ would be a smaller counterexample, and if $\widetilde{x}_{u}=1, u$ has a neighbor $v$ with $\widetilde{x}_{v}=0$.

$\widetilde{x}$ is the unique solution of the system

$$
\begin{aligned}
x_{u}+x_{v} & = & 1 & \\
\sum_{u \in V(C)} x_{u} & =\frac{1}{2}(|V(C)|-1) & & (C \in \mathcal{C}),
\end{aligned}
$$

as otherwise $\widetilde{x}$ would not be a vertex of $\mathcal{P}(\widetilde{G})$. For $V_{0} \subseteq V(\widetilde{G})$, we define $\mathcal{T}\left(V_{0}\right):=$ $\left\{u v \in \mathcal{T} \mid u \in V_{0}\right\}$ and $\mathcal{C}\left(V_{0}\right):=\left\{C \in \mathcal{C} \mid V(C) \cap V_{0} \neq \emptyset\right\}$.

$$
\text { For each } V_{0} \varsubsetneqq V(\widetilde{G}):\left|\mathcal{T}\left(V_{0}\right)\right|+\left|\mathcal{C}\left(V_{0}\right)\right|>\left|V_{0}\right|
$$

If this were not true, the restriction of $\widetilde{x}$ to $V(\widetilde{G}) \backslash V_{0}$ would be a unique solution of the system

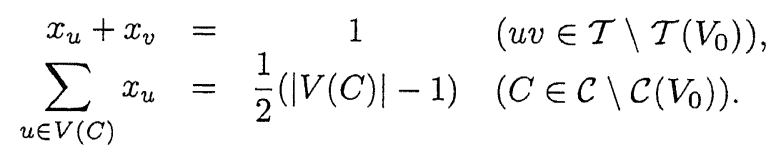

So $\widetilde{G} \backslash V_{0}$ would be a smaller counterexample to Theorem 1. From (14), it also follows that

$$
\delta(v) \nsubseteq \mathcal{T} \text { for each } v \in V
$$

ClaIM 15. If $C$ is an odd circuit, then $E(C) \cap \mathcal{T}$ contains no matching of size $\frac{1}{2}(|V(C)|-1)$. If $C$ is an even circuit and $E(C) \cap \mathcal{T}$ contains a perfect matching, then $E(C) \subseteq \mathcal{T}$.

Proof of Claim 15. Let $M \subseteq E(C) \cap \mathcal{T}$ be a matching with at least $\frac{1}{2}(|V(C)|-1)$ edges. If $C$ is even, then $\frac{1}{2}|V(C)|=\sum_{u v \in M}\left(\widetilde{x}_{u}+\widetilde{x}_{v}\right)=\sum_{u v \in E(C) \backslash M}\left(\widetilde{x}_{u}+\widetilde{x}_{v}\right) \leq$ $\frac{1}{2}|V(C)|$; thus, we have equality throughout, which implies that also edges in $E(C) \backslash M$ are in $\mathcal{T}$. If $C$ is odd, then there is exactly one node $u^{\prime} \in V(C)$ that is incident 
with none of the edges in $M$, so we have $\widetilde{x}_{u^{\prime}}=\sum_{v \in V(C)} x_{v}-\sum_{u v \in M}\left(x_{u}+x_{v}\right) \leq$ $\frac{1}{2}(|V(C)|-1)-\frac{1}{2}(|V(C)|-1)=0$, which contradicts (16).

ClaIM 16. Let $u$ and $v$ be two nodes on a circuit $C \in \mathcal{C}$ and $P$ be a uv-path that is internally node disjoint from $C$. If $\mathcal{T} \cap E(P)$ contains a matching $M$ covering each node in $V(P) \backslash\{u, v\}$, then the unique odd circuit in $C \cup P$ using $P$ is tight.

Proof of Claim 16. Let $Q_{1}$ and $Q_{2}$ be the two uv-paths in $C$, and assume that $P \cup Q_{1}$ is an odd circuit- $C^{\prime}$, say. Let $N$ be the largest matching in $E\left(Q_{2}\right)$ with $V(N) \cap\{u, v\}=V(M) \cap\{u, v\}$. Then $\sum_{r \in V\left(C^{\prime}\right)} \widetilde{x}_{r}=\sum_{r \in V\left(C^{\prime}\right) \backslash V(M)} \widetilde{x}_{r}+\sum_{r s \in M}\left(\widetilde{x}_{r}+\right.$ $\left.\widetilde{x}_{s}\right)=\sum_{r \in V(C) \backslash V(N)} \widetilde{x}_{r}+|M| \geq \sum_{r \in V(C) \backslash V(N)} \widetilde{x}_{r}+\sum_{r s \in N}\left(\widetilde{x}_{r}+\widetilde{x}_{s}\right)-|N|+|M|=$ $\sum_{r \in V(C)} \widetilde{x}_{r}-|N|+|M|=\frac{1}{2}(|V(C)|-1)-|N|+|M|=\frac{1}{2}\left(\left|V\left(C^{\prime}\right)\right|-1\right)$. Thus $C^{\prime} \in \mathcal{C}$.

A circuit $C$ in a graph $G$ is called separating if $G$ has subgraphs $G_{1}$ and $G_{2}$, each properly containing $C$, such that $V(G)=V\left(G_{1}\right) \cup V\left(G_{2}\right), E(G)=E\left(G_{1}\right) \cup E\left(G_{2}\right)$, $V(C)=V\left(G_{1}\right) \cap V\left(G_{2}\right)$, and $E(C)=E\left(G_{1}\right) \cap E\left(G_{2}\right)$.

ClaIm 17. No circuit in $\mathcal{C}$ is separating.

Proof of Claim 17. Let $C \in \mathcal{C}$ be separating, and let $G_{1}$ and $G_{2}$ be as indicated just above this claim (with $G=\widetilde{G}$ ). For $i=1,2$, let $\widetilde{x}^{i}$ be the restriction of $\widetilde{x}$ to $V\left(G_{i}\right)$. As both $G_{1}$ and $G_{2}$ have no bad- $K_{4}$ and fewer edges than $\widetilde{G}$, they are tperfect. Therefore, there exists a $K \in \mathbb{N}$, stable sets $S_{1}^{1}, \ldots, S_{K}^{1}$ in $G_{1}$, and stable sets $S_{1}^{2}, \ldots, S_{K}^{2}$ in $G_{2}$ (with possible repetitions) such that

$$
\widetilde{x}^{1}=\frac{1}{K}\left(\chi_{S_{1}^{1}}+\cdots+\chi_{S_{K}^{1}}\right) \operatorname{and} \widetilde{x}^{2}=\frac{1}{K}\left(\chi_{S_{1}^{2}}+\cdots+\chi_{S_{K}^{2}}\right) .
$$

Consequently,

$$
\left|S_{j}^{i} \cap V(C)\right|=\frac{1}{2}(|V(C)|-1) \text { for } i=1,2 \text { and } j=1, \ldots, K .
$$

For $i=1,2$ and $u v \in E(C)$, we denote the number of stable sets $S_{j}^{i}$ with $u, v \notin S_{j}^{i}$ by $\sigma_{i}(u v)$. As, $\sigma_{i}(u v)=\sum_{j=1}^{K}\left(1-\left|S_{j}^{i} \cap\{u, v\}\right|\right)=K-\sum_{j=1}^{K} \chi_{\{u, v\}}^{\top} \chi_{S_{j}^{i}}=K-K \chi_{\{u, v\}}^{\top} \widetilde{x}^{i}=$ $K\left(1-\widetilde{x}_{u}^{i}-\widetilde{x}_{v}^{i}\right)=K\left(1-\widetilde{x}_{u}-\widetilde{x}_{v}\right)$, we have that

$$
\sigma_{1}(u v)=\sigma_{2}(u v) \text { for each } u v \in E(C) .
$$

By (21) and (22), we can renumber the sets $S_{1}^{2}, \ldots, S_{K}^{2}$, such that

$$
\text { for all } j=1, \ldots, K, S_{j}^{1} \cap V(C)=S_{j}^{2} \cap V(C) \text {. }
$$

Hence, each $S_{j}^{1} \cup S_{j}^{2}$ is a stable set in $\widetilde{G}$ and

$$
\widetilde{x}=\frac{1}{K}\left(\chi_{S_{1}^{1} \cup S_{1}^{2}}+\cdots+\chi_{S_{K}^{1} \cup S_{K}^{2}}\right),
$$

but this contradicts that $\widetilde{x}$ is a fractional vertex of $\mathcal{P}(\widetilde{G})$.

As $\widetilde{G}$ is not t-perfect, it contains an odd- $K_{4}$. So, by (15) and Theorem $2, \widetilde{G}$ is an odd- $P_{9}$, a book or a clean pad. We will deal with these cases separately.

CASE 1. $\widetilde{G}$ is an odd-P.

By (15), $\widetilde{G}$ is in fact the Petersen graph with a node removed; see Figure 13. Let $S_{3,6}=\left\{u_{3}, u_{6}, u_{14}, u_{25}\right\}$. By (17), there exists an edge $u v \in \mathcal{T}$ with $S_{3,6} \cap\{u, v\}=\emptyset$ or a $C \in \mathcal{C}$ such that $\left|S_{3,6} \cap V(C)\right|<(|V(C)|-1) / 2$. It is easy to check that the only 


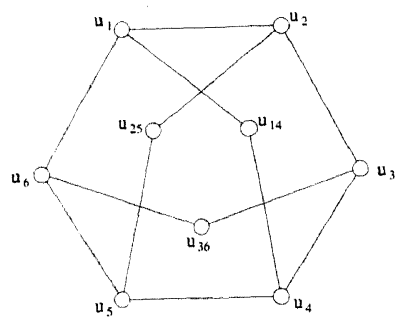

FIG. 13.

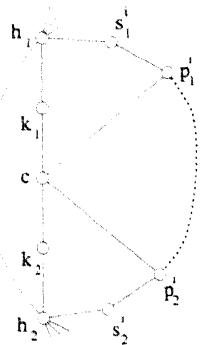

(a)

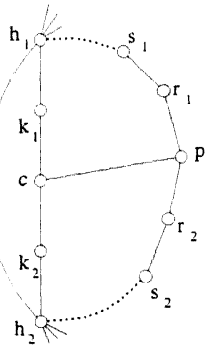

(b)

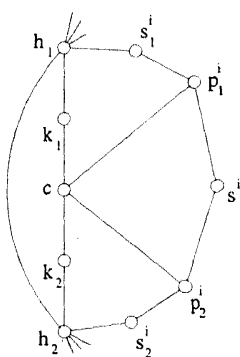

(c)

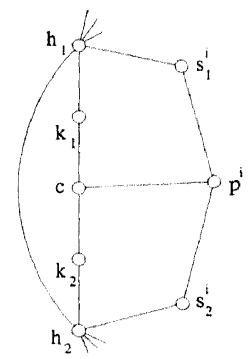

(d)

FIG. 14. Dotted curves indicated internally node disjoint even paths; they may have length zero.

possibility for this to hold is that either $u_{1} u_{2} \in \mathcal{T}$ or $u_{5} u_{4} \in \mathcal{T}$. By symmetry, we also have $u_{2} u_{3} \in \mathcal{T}$ or $u_{6} u_{5} \in \mathcal{T}$ and $u_{3} u_{4} \in \mathcal{T}$ or $u_{6} u_{1} \in \mathcal{T}$. Again by symmetry, we may assume that $u_{1} u_{2} \in \mathcal{T}$. Hence by Claim 15, $u_{6} u_{5} \notin \mathcal{T}$ and $u_{3} u_{4} \notin \mathcal{T}$. So $u_{6} u_{1} \in \mathcal{T}$ and $u_{2} u_{3} \in \mathcal{T}$. However, that contradicts Claim 15 .

CASE 2. $\widetilde{G}$ is a book.

Let $h_{1}, h_{2}$ be the hinges of the book and $c$ be the center. Let $L_{1}, \ldots, L_{n}$ be the trims of the book. By (15), the spine of $\widetilde{G}$ is a circuit of length $5-h_{1} k_{1} c k_{2} h_{2}$, sayand the legs of each $L_{i}$ have length two. Let $h_{1} s_{1}^{i} p_{1}^{i}$ be the first leg of $L_{i}$ and $p_{2}^{i} s_{2}^{i} h_{2}$ be the last leg of $L_{i}$ (going from $h_{1}$ to $h_{2}$; see Figure 14a). It is straightforward to check that

(25) each nonseparating odd circuit in $\widetilde{G}$ is one of $h_{1} h_{2} \cup L_{i} ; h_{1} s_{1}^{i} p_{1}^{i} c k_{1}$ or $h_{2} s_{2}^{i} p_{2}^{i} c k_{2}$ for some $i=1, \ldots, n$.

CLAIM 18. If $p \in L_{i}$ and $c p \in E(\widetilde{G})$, then $|\mathcal{C}(p)| \geq 2$. Hence $p \in\left\{p_{1}^{i}, p_{2}^{i}\right\}$.

Proof of Claim 18. Assume $|\mathcal{C}(p)| \leq 1$. Let $s_{1} r_{1} p$ and $p r_{2} s_{2}$ be the two legs of $L_{i}$ adjacent to $p$; see Figure $14 \mathrm{~b}$. By $(18),\left|\mathcal{T}\left(r_{1}, p, r_{2}\right)\right| \geq 4-\left|\mathcal{C}\left(r_{1}, p, r_{2}\right)\right| \geq 3$. By (18) and $(19),\left|\mathcal{T}\left(r_{1}\right)\right|=\left|\mathcal{T}\left(r_{2}\right)\right|=1$. Hence $c p \in \mathcal{T}$. By (19), we may assume that $p r_{1} \notin \mathcal{T}$; hence $r_{1} s_{1} \in \mathcal{T}$. But now the circuit $c p r_{1} s_{1}$ or, if $s_{1}=h_{1}$, the circuit $c p r_{1} s_{1} k_{1}$ violates Claim 15.

Claim 19. For each $i=1, \ldots, n, p_{1}^{i}=p_{2}^{i}=: p^{i}$ (see Figure $14 \mathrm{~d}$ ).

Proof of Claim 19. If not, $L_{i}$ has three legs; see Figure 14c. By (19), (18), and (25), $\left|\mathcal{T}\left(s^{i}\right)\right|=1$, so, by symmetry, we may assume that $s^{i} p_{2}^{i} \in \mathcal{T}$. By Claim 18 , the circuit $c k_{1} h_{1} s_{1}^{i} p_{1}^{i}$ is tight, so by Claim $16, c k_{1} h_{1} s_{1}^{i} p_{1}^{i} s^{i} p_{2}^{i}$ is tight as well. But it has a chord, contradicting Claim 17.

Claim 20. $c k_{1}, c k_{2} \in \mathcal{T}$.

Proof of Claim 20. Suppose $c k_{2} \notin \mathcal{T}$. Let $S:=\left\{k_{1}, h_{2}\right\} \cup\left\{p^{i} \mid 1=i, \ldots, n\right\}$. 
By (17), there exists an edge $u v \in \mathcal{T}$ with $S \cap\{u, v\}=\emptyset$ or an odd circuit $C \in \mathcal{C}$ with $|S \cap V(C)|<\frac{1}{2}(|V(C)|-1)$. Using (25), it is easy to check that this implies that $h_{1} s_{1}^{i} \in \mathcal{T}$ for some $i=1, \ldots, n$. Fix such an $i$. By (19) and Claim 15, none of $s_{1}^{i} p^{i}, p^{i} c, p^{i} s_{2}^{i}$, and $s_{2}^{i} h_{2}$ is in $\mathcal{T}$. By (18), $\left|\mathcal{C}\left(s_{1}^{i}, p^{i}, s_{2}^{i}\right)\right| \geq 4-\left|\mathcal{T}\left(s_{1}^{i}, p^{i}, s_{2}^{i}\right)\right|=3$, so, $c k_{2} h_{2} s_{2}^{i} p^{i} \in \mathcal{C}$ and $s_{1}^{i} h_{1} h_{2} s_{2}^{i} p^{i} \in \mathcal{C}$. Hence $\widetilde{x}_{c}+\widetilde{x}_{k_{2}}=\widetilde{x}_{s_{1}^{i}}+\widetilde{x}_{h_{1}}$, contradicting that $s_{1}^{i} h_{1}$ is tight and $c k_{2}$ is not.

Now, by (19), we may assume that $c p^{1} \notin \mathcal{T}$. By Claims 20 and $15, \mathcal{T}\left(s_{1}^{1}\right)=$ $\mathcal{T}\left(s_{1}^{2}\right)=\emptyset$. Hence $\left|\mathcal{T}\left(s_{1}^{1}, p^{1}, s_{2}^{1}\right)\right|+\left|\mathcal{C}\left(s_{1}^{1}, p^{1}, s_{2}^{1}\right)\right| \leq 3$, contradicting (18).

CASE $3 . \widetilde{G}$ is a clean pad.

A priori, the tight odd circuits might run quite wildly through $\widetilde{G}$. However, this is not the case, as is shown by the following lemma, which can be understood independently of the present proof.

LEMMA 13. Let $C$ be a nonseparating odd circuit in a clean pad $G$. Then $\mid E(C) \cap$ $K(G) \mid=1$.

Proof. Let $G$ be a counterexample with $|E(G)|$ minimal. Let $C$ be a nonseparating odd circuit in $G$ with $|E(C) \cap K(G)| \neq 1$. As contracting all edges on $E(C) \cap R(G)$ yields another counterexample, $E(C) \subseteq K(G)$. Moreover, if $e \in K(G) \backslash E(C)$, then its end nodes lie in different components of $R(G) \backslash V(C)$, as otherwise, $G \backslash\{e\}$ would be a smaller counterexample. We first prove that

$$
E(C) \text { contains no pair of parallel chords. }
$$

Indeed, suppose that it is false. Choose parallel chords $f, g \in E(C)$ that are distant in the pad $G \backslash(K(G) \backslash E(C))$. As $C$ is nonseparating, there exist edges $e_{f}, e_{g} \in$ $K(G) \backslash E(C)$ with no end node in $V(C)$ such that $e_{f} \times f$ and $e_{g} \times g$. If $e_{f} \| g$ and $e_{g} \| f$, then $G$ is not clean. Thus, we may assume $e_{f} \times g$. As $C$ is odd, not all edges on $C$ can cross $e_{f}$, so there exists an $h \| e_{f}$, but then, as $f$ and $g$ are distant in the pad $G \backslash(K(G) \backslash E(C))$, the chords $e_{f}, f, g$, and $h$ form a mesh.

Let $c_{0}, \ldots, c_{2 k}$ be the nodes of $C$, numbered in the order in which they lie around $R(G)$. From (26) it then follows that the edges of $C$ are $c_{i} c_{i+k}$ (indices modulo $2 k+1)$. Let $P_{i}$ be the $c_{i} c_{i+1}$-path on $R(G)$ that contains no nodes of $C$ other than $c_{i}$ and $c_{i+1}$, see Figure 14a. Let $K_{i}:=\left\{u v \in K(G) \backslash E(C) \mid u \in V\left(P_{i}\right)\right\}$; note that for each $i=0, \ldots, 2 k, K_{i} \neq \emptyset$. For each $e \in K(G) \backslash E(C)$ let $C_{e}$ be the odd circuit in $R(G) \cup\{e\}$ that uses the fewest nodes of $V(C)$.

$$
\text { If } e, f \in K_{i} \text {, then } V\left(C_{e}\right) \cap\left\{c_{i}, c_{i+1}\right\}=V\left(C_{f}\right) \cap\left\{c_{i}, c_{i+1}\right\} \text {. }
$$

Indeed, if not, $c_{i} c_{i+k+1}, c_{i+1} c_{i+k+1}, e$, and $f$ show that $G$ is nonnesting or has a mesh, and hence is not clean.

From (27), it is easy to see that there exists an $i=0, \ldots, 2 k$, such that $V\left(C_{e}\right) \ni c_{i}$ for all $e \in K_{i} \cup K_{i-1}$. By circular symmetry, we may assume that $k+1$ is such an $i$. Let $f \in K_{0}$. By the symmetry $i \leftrightarrow 2 k+2-i(\bmod 2 k+1)$, we may assume that $c_{1} \in V\left(C_{f}\right)$; hence $f \| c_{0} c_{k+1}$ and $f \times c_{1} c_{k+2}$. Let $e \in K_{k+1}$. Then $e \| c_{1} c_{k+2}$ and $e \times c_{0} c_{k+1}$. Hence $c_{0} c_{k+1}, c_{1} c_{k+2}, e$, and $f$ form a mesh (see Figure 14b). $\quad$

For each $C \in \mathcal{C}$, we denote the unique edge in $E(C) \cap K(\widetilde{G})$ by $k[C]$. Our next task is to study the structure of the collection of tight edges and odd circuits as a whole. The outcome will be summarized in (35), (36), and (37); for proving those we need to derive some claims. We define, for each $\ell=0,1, \ldots$, $K_{\ell}:=\{e \in K(\widetilde{G}) \mid e$ is in $\ell$ tight odd circuits $\}, K_{\ell}^{\text {tight }}:=K_{\ell} \cap \mathcal{T}$, and $K_{\ell}^{\text {free }}:=K_{\ell} \backslash \mathcal{T}$. By Lemma $13, K_{\ell}=\emptyset$ for $\ell \geq 3$. Moreover,

$$
K_{0}^{\text {free }}=\emptyset
$$




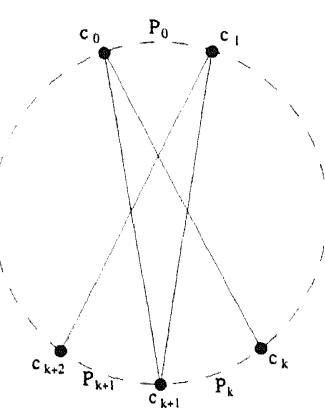

(a)

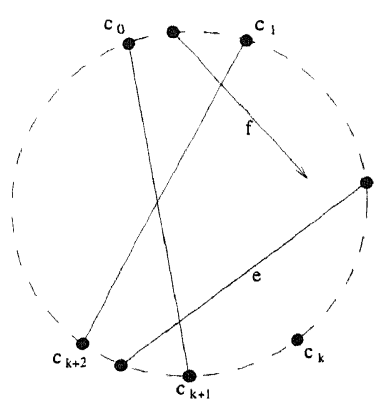

(b)

FIG. 15. Dashed curves indicate internally node disjoint even paths of positive length. The closed curve on the outside is the rim.

as deleting an edge in $K_{0}^{\text {free }}$ form $\widetilde{G}$ would yield a smaller non-t-perfect graph.

ClaIM 21. If $u v \in K(\widetilde{G}) \cap \mathcal{T}$, then uv is the only chord with end node $u$.

Proof of Claim 21. Let $u w$ be a second chord. Let $P:=v v^{\prime} \ldots w^{\prime} w$ be the $v w$-path on $R(\widetilde{G})$ not containing $u$. If there exists a tight odd circuit using both $v$ and $w$, then by Claim 16, there exists a tight odd circuit using $v u$ and $u w$, but this contradicts Claim 17 or Lemma 13. Let $C_{w} \in \mathcal{C}\left(w^{\prime}\right)$ and $C_{v} \in \mathcal{C}\left(v^{\prime}\right)$. By Claim 17, $u \notin V\left(C_{w}\right) \cup V\left(C_{v}\right)$, so $k\left[C_{w}\right]$ crosses $u w$ and $k\left[C_{v}\right]$ crosses $u v$. Hence, $u w, u v, k\left[C_{w}\right]$, and $k\left[C_{v}\right]$ show that $\widetilde{G}$ has a mesh or is nonnesting-a contradiction!

Claim 22. If $u v \in K(\widetilde{G}) \cap \mathcal{T}$, then uv is not a universal chord of $\widetilde{G}$.

Proof of Claim 22. Suppose that the claim is false. We construct a new graph $G$ from $\widetilde{G}$ as follows. For each neighbor $w$ of $u$, we introduce a new node $w^{*}$ and two new edges $u w^{*}$ and $w^{*} w$ and remove the original edge $u w$. Moreover, we define $x \in \mathbb{R}^{V(G)}$ by $x_{w}:=\widetilde{x}_{w}$ if $w \in V(\widetilde{G}) \backslash\{u\}, x_{w^{*}}:=\widetilde{x}_{u}$ if $w$ is a neighbor of $u$ in $\widetilde{G}$, and $x_{u}:=1-\tilde{x}_{u}$. Then, by $(14), x$ is a vertex of $P(G)$.

Let $G^{\prime}$ be obtained from $G$ by contracting $u v^{*}$ and $v^{*} v$ into one new node, called $v$ again. As $x_{u}+x_{v^{*}}=1=x_{v^{*}}+x_{v}$, we get from (14) that $G^{\prime}$ is not t-perfect. On the other hand, as $u v$ is universal in $\widetilde{G}$, each odd circuit in $G^{\prime}$ goes through $v$. However, Fonlupt and Uhry [5] have proved that graphs containing a node that lies on each odd circuit are t-perfect-a contradiction.

As tight odd circuits have no chords, we have by Claim 21 and (28) that

$$
|\delta(u) \cap K(\widetilde{G})| \leq 2 \text { for all } u \in V(\widetilde{G})
$$

and

$$
\text { if } e \in K_{2} \text {, then all other chords cross } e \text {. }
$$

By (30) and Claim 22,

$$
K_{2}^{\mathrm{tight}}=\emptyset .
$$

For each $e \in K(\widetilde{G})$ define $y_{e}$ to be the total number of tight odd circuits and edges containing $e$. From Claims 21 and 22 and by (29) and (30), we see that

$$
\sum_{y_{e}} y_{e} \text { for each } u \in U(\widetilde{G}) \text {. }
$$


Moreover, by (19),

$$
|\mathcal{T}(u)| \leq 1 \text { for each } u \in W(\widetilde{G})
$$

and thus, by (17),

$$
\begin{aligned}
|V(\widetilde{G})| & =|U(\widetilde{G})|+|W(\widetilde{G})| \\
& \geq \frac{1}{2} \sum_{u \in U(\widetilde{G})} \sum_{e \in \delta(u) \cap K(\widetilde{G})} y_{e}+\sum_{u \in W(\widetilde{G})}|\mathcal{T}(u)| \\
& =\sum_{e \in K(\widetilde{G})} y_{e}+\sum_{u \in W(\widetilde{G})}|\mathcal{T}(u)| \\
& =|\mathcal{C}|+|\mathcal{T}| \\
& \geq|V(\widetilde{G})| .
\end{aligned}
$$

Thus, we have equality throughout, which implies that we have equality in (32) and (33). So we get

$$
|\mathcal{T}(u)|=1 \text { for each } u \in W(\widetilde{G})
$$

(36) each chord in $K_{1}^{\text {tight }} \cup K_{2}^{\text {free }}$ is node disjoint from all other chords; moreover, the edges in $K_{1}^{\text {free }}$ form node disjoint circuits;

and, by Claim 22,

$$
K_{0}^{\text {tight }}=\emptyset
$$

As $W(\widetilde{G})$ is a stable set, by (17), there exists an equation in (17) that does not hold for $\chi_{W(\widetilde{G})}$. Case checking yields that this means that

$$
K_{1}^{\mathrm{tight}} \neq \emptyset
$$

Claim 23. $K_{1}^{\text {free }} \neq \emptyset$.

Proof of Claim 23. Suppose that $K_{1}^{\text {free }}=\emptyset$. Then, by (36), no two chords touch. By (38), there exists at least one tight chord, so, by Claim 22, there exists a pair of parallel chords. Choose $e, f \in K(\widetilde{G})$ parallel, such that the shortest path-Q, say-on $R(\widetilde{G})$ that connects an end node of $e$ with an end node of $f$ is as short as possible. Let $C_{e} \in \mathcal{C}(e)$ and $C_{f} \in \mathcal{C}(f)$ (as $e$ and $f$ are parallel, these two odd circuits are unique). Let $u \in W(G) \cap V(Q)$ and $C \in \mathcal{C}(u)$. (C exists by (18) and (35).) As $u \notin V\left(C_{e}\right) \cup V\left(C_{f}\right), e, f \neq k[C]$, and by the choice of $e$ and $f, k[C]$ is not parallel to $e$ nor to $f$. Therefore, as there are no touching chords, $k[C]$ crosses both $e$ and $f$. As $k[C]$ is tight, there exists an edge $h \| k[C]$. As $h$ is not a chord of $C_{e}$ nor of $C_{f}$, $k[C], e, f$, and $h$ form a mesh-a contradiction!

For each $e \in K_{1}$ let $C[e]$ be the unique tight odd circuit using $e$.

ClaIm 24. $K_{1}^{\text {free }}$ contains no pair of parallel chords.

Proof of Claim 24. Let $f_{1}=u_{1} u_{2}$ and $f_{2}=v_{1} v_{2}$ be two parallel chords in $K_{1}^{\text {free; }}$; see Figure 15 . Let $P$ be the $u_{2} v_{1}$-path on $R(\widetilde{G})$ containing $v_{2}$. By symmetry we may

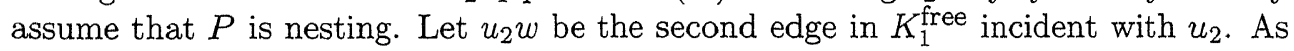




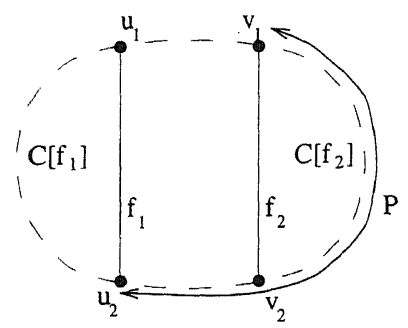

FIG. 16.

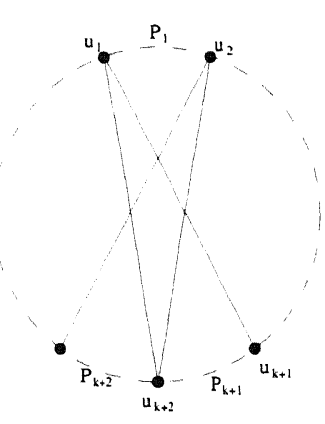

(a)

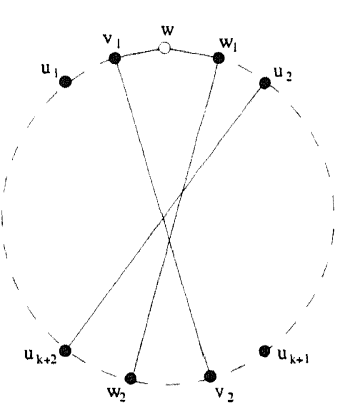

(b)

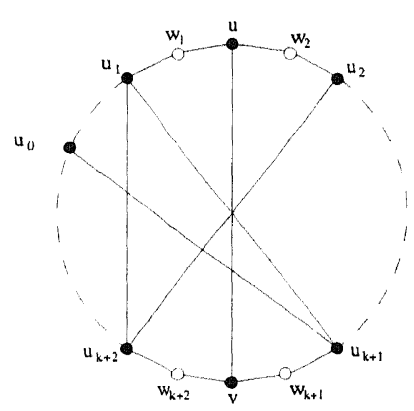

(c)

FIG. 17. Dashed curves indicate internally node disjoint even paths of positive length. The closed curve on the outside is the rim.

$P$ is nesting, $w \notin P \backslash\left\{v_{1}\right\}$, but then either $u_{1} u_{2}$ or $v_{1} v_{2}$ is a chord of $C\left[u_{2} w\right]$, or $u_{2} w$ is a chord of $C\left[u_{1} u_{2}\right]$ - a contradiction!

Let $\Gamma$ be a circuit in $K_{1}^{\text {free }}$. Let $u_{0}, \ldots, u_{N}$ be the nodes of $\Gamma$ in the order in which they lie around $R(\widetilde{G})$. From Claim 24 , it follows that $N$ is even ( $2 k$, say) and that the edges in $\Gamma$ are of the form $u_{i} u_{i+k+1}$ (indices modulo $2 k+1$ ); see Figure 17a. All chords not in $\Gamma$ are parallel with at least one edge in $\Gamma$. Thus, by (28), (30), and Claim 24, we have that

$$
K_{2}=\emptyset \text { and } K_{1}^{\text {free }}=E(\Gamma)
$$

For $i=0, \ldots, 2 k$, let $P_{i}$ be the $u_{i} u_{i+1}$-path on $R(\widetilde{G})$ that is internally node disjoint from $\Gamma$. By (38), there exists an edge $u v$ in $K_{1}^{\text {tight }}$. By symmetry we may assume that $u \in P_{1}$ and $v \in P_{1} \cup \cdots \cup P_{k+1}$. As $C\left[u_{1} u_{k+1}\right]$ has no chords, we have that

$$
v \in P_{k+1} \text {. }
$$

Claim 25. Each chord in $K_{1}^{\text {tight }}$ has one end node in $P_{1}$ and one in $P_{k+1}$.

Proof of Claim 25. Let $x y \in K_{1}^{\text {tight }} \backslash\{u v\}$. As we proved for $u v$, we may assume that $x \in P_{i}$ and $y \in P_{i+k}$. Hence, $u v \| u_{1} u_{k+2}$ and $x y \| u_{i} u_{k+i+1}$. If $i$ were different from 1 , then $x y, u v, u_{1} u_{k+2}$ and $u_{i} u_{k+i+1}$ would form a mesh or show that $\widetilde{G}$ is nonnesting. Hence $i=1$ and the claim follows.

Claim 26. $\left|K_{1}^{\text {tight }}\right|=1$.

Proof of Claim 26. Suppose not; then there are chords $v_{1} v_{2}$ and $w_{1} w_{2}$ in $K_{1}^{\text {tight }}$, such that $u_{1}$ and $w_{1}$ are both on $P_{1}$ and share a common neighbor $w$ on $P_{1}$, see Figure $17 \mathrm{~b}$. From (35) we may assume that $v_{1} w \in \mathcal{T}$, but now the path $v_{2} v_{1} w w_{1} w_{2}$ 
and the circuit $C\left[u_{2} u_{k+2}\right]$ satisfy the assumptions in Claim 16. Hence there exists a tight odd circuit using both $v_{1} v_{2}$ and $w_{1} w_{2}$, contradicting Claim 17 or Lemma 13.

Hence $P_{1}$ and $P_{k+1}$ are paths of length 4 . Let $P_{1}=u_{1} w_{1} u w_{2} u_{2}$ and $P_{2}=$ $u_{k+1} w_{k+1} v w_{k+2} u_{k+2}$; see Figure 17c.

We have that $w_{2} u_{2} \notin \mathcal{T}$, as otherwise the path $v u w_{2} u_{2} u_{k+2}$ and the circuit $C\left[u_{0} u_{k+1}\right]$ would satisfy the assumptions of Claim 16 and thus yield a tight odd circuit using three chords of $\widetilde{G}$. By symmetry also $u_{1} w_{1} \notin \mathcal{T}$. Hence, by $(35), u w_{1}, u w_{2} \in \mathcal{T}$. But as $u v \in \mathcal{T}$ this contradicts (19). This completes the proof of Case 3 and thus of Theorem 1.

Acknowledgments. This research was initiated at the Fourth Bellairs Workshop on Combinatorial Optimization in 1993. The authors sincerely thank the referees for their valuable comments.

\section{REFERENCES}

[1] F. Barahona And A. R. Mahjoub, Compositions of graphs and polyhedra II: Stable sets, SIAM J. Discrete Math., 7 (1994), pp. 359-371.

[2] M. Boulala And J. P. UhrY, Polytope des indépendants d'un graphe série-parallèle, Discrete Math., 27 (1979), pp. 225-243.

[3] P. A. CATLIN, Hajós graph coloring conjecture: Variations and counterexamples, J. Combin. Theory Ser. B, 26 (1979), pp. 268-274.

[4] V. CHVÁtal, Edmonds polytopes and a hierarchy of combinatorial problems, Discrete Math., 4 (1975), pp. 305-337.

[5] J. FONLUPT AND J. P. UHRY, Transformations which preserve perfection and h-perfection of graphs, Ann. Discrete Math., 16 (1982), pp. 83-95.

[6] A. M. H. Gerards, A min-max relation for stable sets in graphs with no odd- $K_{4}$, J. Combin. Theory Ser. B, 47 (1989), pp. 330-348.

[7] A. M. H. Gerards, Graphs and Polyhedra-Binary Spaces and Cutting Planes, CWI Tract 73, CWI, Amsterdam, 1990.

[8] A. M. H. Gerards, L. Lovász, A. Schrijver, P. D. Seymour, C.-H. Shih, and K. Truemper, Regular matroids from graphs, in preparation.

[9] A. M. H. Gerards AND A. SchriJver, Matrices with the Edmonds-Johnson property, Combinatorica, 6 (1986), pp. 365-379.

[10] N. SBihi And J. P. UhrY, A class of h-perfect graphs, Discrete Math., 51 (1984), pp. 191-205.

[11] F. B. Shepherd, Applying Lehman's theorems to packing problems, Math. Programming, 71 (1995), pp. 353-367.

[12] B. TofT, Problem 10, in Recent Advances in Graph Theory: Proc. Symposium Prague, June 1974, M. Fiedler, ed., Academia, Praha, 1975, pp. 543-544. 